\title{
Ensuring Trust Of Third-Party Hardware Design With Constrained Sequential Equivalence Checking
}

\author{
Gyanendra Shrestha \\ Thesis submitted to the Faculty of the \\ Virginia Polytechnic Institute and State University \\ in partial fulfillment of the requirements for the degree of \\ Master of Science \\ in \\ Computer Engineering \\ Michael S. Hsiao, Chair \\ Chao Wang \\ Patrick Schaumont \\ September 14, 2012 \\ Blacksburg, Virginia
}

Keywords: Hardware Trojan, RTL, Miter, BMC

Copyright 2012, Gyanendra Shrestha 


\title{
Ensuring Trust Of Third-Party Hardware Design With Constrained Sequential Equivalence Checking
}

Gyanendra Shrestha

\author{
(ABSTRACT)
}

Globalization of semiconductor design and manufacturing has led to a concern of trust in the final product. The components may now be designed and manufactured from anywhere in the world without the direct supervision of the buyer. As a result, the hardware designs and fabricated chips may be vulnerable to malicious alterations by an adversary at any stage of VLSI design flow, thus compromising the integrity of the component. The effect of any modifications made by the adversary can be catastrophic in the critical applications. Because of the stealthy nature of such insertions, it is extremely difficult to detect them using traditional testing and verification methods. Therefore, the trust of the hardware systems require a new approach and have drawn much attention in the hardware security community.

For many years, the researchers have developed sophisticated techniques to detect, isolate and prevent malicious attacks in cyber security community assuming that the underlying hardware platform is extremely secure and trustworthy. But the hardware may contain one or more backdoors that can be exploited by software at the time of operation. Therefore, the trust of the computing system cannot be guaranteed unless we can guarantee the trust of the hardware platform.

A malicious insertion can be very stealthy and may only involve minor modification in the 
hardware design or the fabricated chip. The insertion may require rare or specific conditions in order to be activated. The effect may be denial of service, change of function, destruction of chip, leakage of secret information from cryptographic hardware etc.

In this thesis, we propose a novel technique for the detection of malicious alteration(s) in a third party soft intellectual property (IP) using a clever combination of sequential equivalence checking (SEC) and automatic test generation. The use of powerful inductive invariants can prune a large illegal state space, and test generation helps to provide a sensitization path for nodes of interest. Results for a set of hard-to-verify designs show that our method can either ensure that the suspect design is free from the functional effect of any malicious change(s) or return a small group of most likely malicious signals.

This material is based upon work supported by the National Science Foundation under Grant No. 1134843. Any opinions, findings, and conclusions or recommendations expressed in this material are those of the author(s) and do not necessarily reflect the views of the National Science Foundation. 


\section{Dedication}

I dedicate this thesis to my grandfather, my parents, my wife and my brother. 


\section{Acknowledgements}

I extend my gratitude to my advisor Prof. Dr. Michael S. Hsiao for his consistent guidance and continuous support throughout my research. His suggestions and encouragements were extremely valuable and productive to help me complete my thesis. I would like to convey my warmest thanks to all the members of PROACTIVE Lab whose help and support were very beneficial to this thesis. I sincerely thank all my friends including Kiran Adhikari, Supratik Mishra, Sarvesh Prabhu, Avinash Desai, Kavya Shagrithaya and Sanjay Basnet for their direct and indirect support.

I would also like to convey my thanks to my beloved grandfather, my parents, my wife Ramita Shrestha and all other family members for their endless support.

Gyanendra Shrestha

September 14, 2012 


\section{Contents}

1 Introduction $\quad 1$

1.1 Hardware Trojans . . . . . . . . . . . . . . . . . . 2

1.2 Hardware Security Measures . . . . . . . . . . . . . . . . . 4

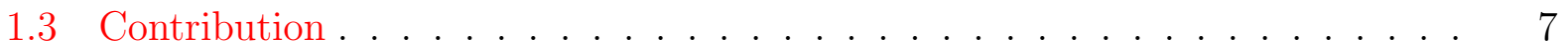

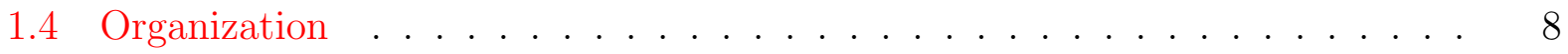

2 Background $\quad 9$

2.1 Trojan Characteristics and Classification . . . . . . . . . . . 9

2.2 Previous Works . . . . . . . . . . . . . . . . . . . 14

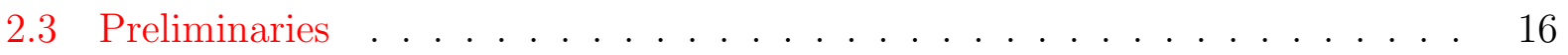

2.3.1 Suspicious Signals . . . . . . . . . . . . . . . . 16

2.3.2 Path Sensitization ...................... 17 
2.3 .3 Static Logic Implication $\ldots \ldots \ldots$

2.3.4 Boolean Satisfiability (SAT) and Conjunctive Normal Form . . . . . . 22

2.3.5 Model Checking . . . . . . . . . . . . . . . . . . . . . . 25

2.3.6 Equivalence Checking and Miter Circuit . . . . . . . . . . . 27

2.3 .7 Invariants . . . . . . . . . . . . . . . . . 30

2.3.8 Random Simulation and Potential Invariants Generation . . . . . . 31

2.3.9 Proving Invariants and Acceleration Techniques . . . . . . . . . . 34

2.3.10 Sequential Equivalence Checking Framework . . . . . . . . . . . 37

3 Test Generation and Equivalence Checking 41

3.1 Motivation . . . . . . . . . . . . . . . . . . . . 42

3.2 Random Simulation and Equivalence Checking . . . . . . . . . . . . . 43

3.2.1 Suspect Signals and Inductive Invariants Generation _. . . . . . . 44

3.2 .2 Application of SEC to the Miter . . . . . . . . . . . . 45

3.3 Detection of Malicious Signals _ . . . . . . . . . . . . . 47

3.3 .1 Invariant-Based Test Generation . . . . . . . . . . . . . . 47

3.3.2 Counterexample Guided Equivalence Checking . . . . . . . . . . . . 54

3.4 Novelty of the Approach . . . . . . . . . . . . . . . . 57 
3.5 Experimental Results . . . . . . . . . . . . . . . . . . . . . 58

3.6 Limitations of the Approach . . . . . . . . . . . . . . . . . . 63

3.7 Summary . . . . . . . . . . . . . . . . . . . . . . . . . 64

4 Strengthened Trojan Detection Method $\quad 65$

4.1 Motivation . . . . . . . . . . . . . . . . 66

4.2 Modified Test Generation . . . . . . . . . . . . . . . . . . . 67

4.3 Relaxed Input Vector as Constraint . . . . . . . . . . . . . . . . . 70

4.4 Ranking of Stealthiness of Malicious Signals . . . . . . . . . . . . . 72

4.5 Experimental Results . . . . . . . . . . . . . . . . . . . . . 74

4.6 Conclusion . . . . . . . . . . . . . . . . . . . . . 76

$\begin{array}{llr}5 & \text { Conclusion and Future Work } & 78\end{array}$

$\begin{array}{lr}\text { Bibliography } & 80\end{array}$ 


\section{List of Figures}

1.1 Levels of trust at different stages of IC design flow. . . . . . . . . . . . 3

2.1 Example of a simple Trojan. . . . . . . . . . . . . . . . . 10

2.2 Trojan Classification. . . . . . . . . . . . . . . . . . 11

2.3 Example for Path Sensitization . . . . . . . . . . . . . . 17

2.4 Example of Direct and Indirect Implications . . . . . . . . . . . . . . . . . 21

2.5 Implication Graph Example . . . . . . . . . . . . . . . . . . . . . 22

2.6 A simple combinational circuit for $\mathrm{CNF}$ conversion . . . . . . . . . . . . . 24

2.7 Miter Circuit . . . . . . . . . . . . . . . . . . . . . . 28

2.8 Unrolled Circuit . . . . . . . . . . . . . . . . . . . . . . 29

2.9 Relationship among invariants . . . . . . . . . . . . . . . 33

2.10 Flowchart for Sequential Equivalence Checking . . . . . . . . . . . . . 38

2.11 Base Case for Sequential Equivalence Checking . . . . . . . . . . . . . . 39 
2.12 Induction Case for Sequential Equivalence Checking . . . . . . . . . . . . . 40

3.1 Miter circuit with suspect and spec circuits . . . . . . . . . . . . . . . . 44

3.2 Flowchart for Invariant based Test Generation . . . . . . . . . . . . . . . . . 49

3.3 Invariant based Test Generation . . . . . . . . . . . . . . . . . . 50

3.4 State space constraining with the addition of $K^{\prime}$ timeframes . . . . . . . 52

3.5 Strengthening the Test Generation Method . . . . . . . . . . . . . . 53

3.6 Flowchart for Counterexample Guided Equivalence Checking . . . . . . . . . 54

3.7 Counterexample guided Equivalence Checking . . . . . . . . . . . . . 55 


\section{List of Tables}

2.1 Table Showing Direct Forward And Backward Implications For Different Gates 20

2.2 Table Showing CNF Formulation For Different Gates . . . . . . . . . . . 23

2.3 Parallel Random Simulation . . . . . . . . . . . . . . . . . . . . . . 32

3.1 Results for Equivalence Checking of Hard SEC Benchmark Circuits . . . . 59

3.2 Results for Malicious Insertion Detection for Hard SEC Benchmark Circuits 61

4.1 Results for Improved Test Generation and Vector Relaxation . . . . . . . . 75 


\title{
List of Abbreviations
}

\author{
ATPG Automatic Test Pattern Generation \\ BDDs Binary Decision Diagrams \\ BMC Bounded Model Checking \\ CAD Computer-Aided Design \\ CGEC Counterexample Guided Equivalence Checking \\ CNF Conjunctive Normal Form \\ CUV Circuit Under Verification \\ DFTT Design for Trojan Test \\ EDA Electronic Design Automation \\ FF $\quad$ Flip-flop \\ FSM Finite State Machine
}




\begin{tabular}{ll} 
HDL & Hardware Description Language \\
IC & Integrated Circuit \\
IP & Intellectual Property \\
LP & Linear Programming \\
PCB & Printed Circuit Board \\
PI & Primary Input \\
PO & Primary Output \\
PPI & Pseudo-Primary Input \\
PPO & Pseudo-Primary Output \\
PUF & Physical Unclonable Function \\
VLSI & Very Large Scale Integration \\
RTL & Register-Transfer Level \\
SAT & Satisfiability \\
SEC & Sequential Equivalence Checking \\
& \\
\hline
\end{tabular}




\section{Chapter 1}

\section{Introduction}

Modern semiconductor industry has been progressing in a rapid pace for the past few decades with the development of various electronic products. With their growing usage in wide variety of areas including education, health, office, industry, military, transportation etc., it has been possible to accommodate a large design with multiple functionalities into a single low-power and high-performance Integrated Circuit (IC) chip. The advancement in Very Large Scale Integration (VLSI) technology and Electronic Design Automation (EDA) tools driven by Moore's law [1], proposed by Gordon Moore in 1965, has resulted in the production of extremely complex, reliable and efficient ICs. After the introduction of open-economy policies by some Asian countries with very low labor costs such as India and China, many companies have migrated some of their IC design and/or fabrication units to such countries in order to reduce overall production costs, utilize wider area of expertise, meet time-tomarket challenges and increase productivity. A complex IC design may include a number 
of offshore Intellectual Property (IP) modules performing different functionalities obtained from third party vendors. However, the inclusion of such IPs and the design, fabrication and assembly of the IC overseas make it vulnerable to malicious alterations by an adversary or a malicious insider at any stage of design flow.

The modifications at different abstraction levels may cause system failure at a critical moment, leak secret key information, destroy the chip, give erroneous output or provide backdoor that acts as killswitch [2] to be exploited by malicious software at the time of operation, thus compromising the integrity of the IC, as well as IC supply chain. The consequence can be catastrophic if the malicious chip is a part of security, safety and mission-critical applications. The trustworthiness of these devices has drawn much attention and concern in recent years in academic research, industries as well as government [2]. Fig. 1.1 shows a typical VLSI design flow with levels of trust at different stages today [3].

\subsection{Hardware Trojans}

Any embedded malicious insertion (also known as Hardware Trojan) is a minute and stealthy alteration in the design made to activate and show its effect under potentially rare (infrequent) events. Generally, a Trojan circuit consists of two parts. The input along with the body of Trojan circuit is called trigger, and the node connected to the output of Trojan circuit which directly gets influenced by its activation effect is called payload. The trigger can be analog and digital circuit (gates, interconnections, physical sensors etc.) whereas 


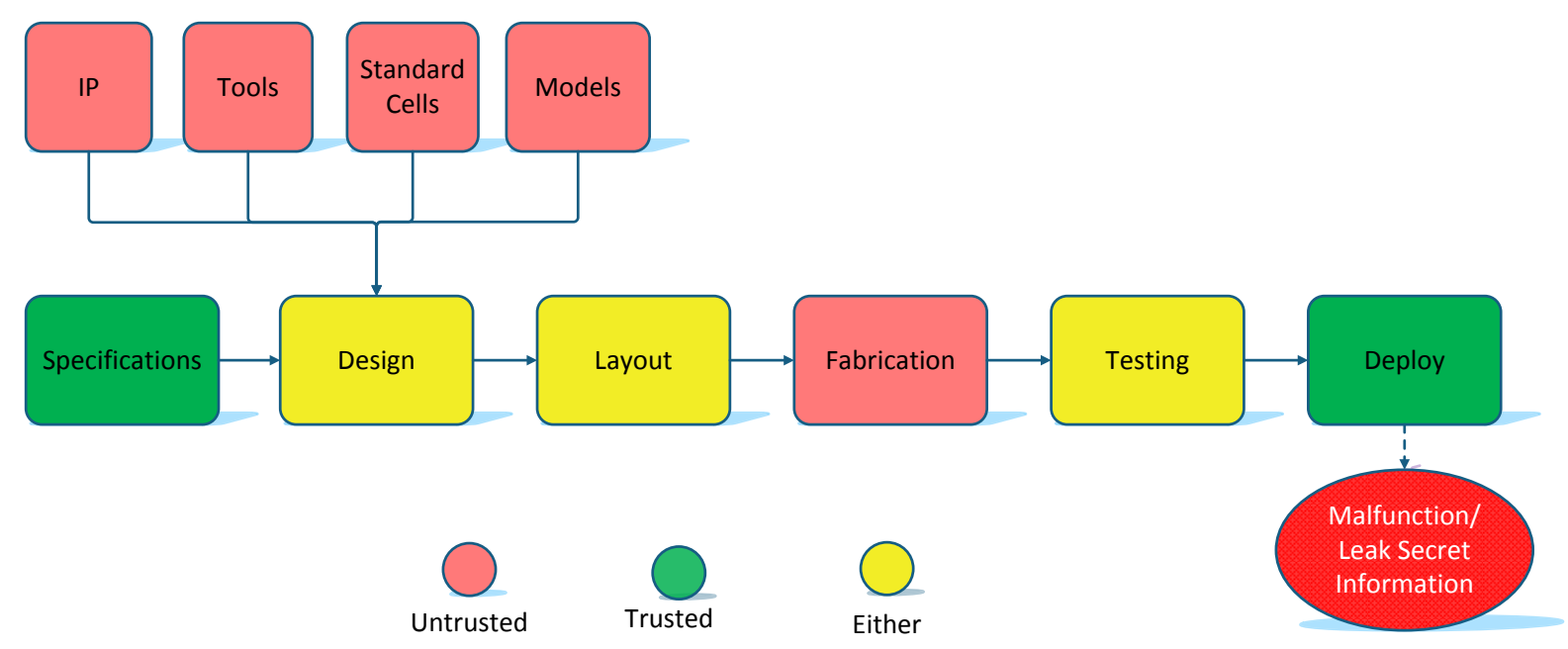

Figure 1.1: Levels of trust at different stages of IC design flow.

Source: DARPA, Trust in Integrated Circuits(TIC), http://www.dtic.mil/cgibin/GetTRD oc?AD=ADA503809, accessed 14 July, 2012. Used under fair use, 2012.

the payload can be a single gate or a minute antenna that can transmit electromagnetic signals. The characteristics of Trojans are different from the anomalous behavior of the chip due to manufacturing defects or functional errors. For instance, a malicious design may perform all the functionalities that the original design does and some extra undesirable functionalities. A Trojan may not change the output behavior at all, but instead leak the secret key information through side-channel power or electromagnetic radiation.

The existence, behavior and activation criteria of Trojans in a third-party IP would be unknown to the system integrator. They can be inserted at any phase of IC development cycle and have different malicious functionalities according to the insertion phase. No matter how secure a software is built, the trust of such software cannot be guaranteed until we guarantee 
the trust of underlying hardware. Catching and detecting the presence of a cleverly designed Trojan can be extremely difficult and time-consuming. This leads to the need of efficient and cost-effective mechanisms to reliably detect and/or prevent the Trojan effects at different untrusted IC design stages.

\subsection{Hardware Security Measures}

Existing hardware security techniques target protection of data in cryptographic hardware devices and wireless networks, and prevention of IP piracy or theft. Researchers have studied several methods of active (e.g. fault injection [4]) and passive (e.g. side-channel analysis) implementation attacks in cryptographic hardware. The most popular side-channel analysis techniques include measurement and analysis of side-channel power consumption statistics, timing information $[5,6]$ and electromagnetic radiation [7]. A number of countermeasures such as time equalization of squaring and multiplication, delay addition [8], power balancing, signal size reduction, noise addition [9] etc. can be applied at different abstraction levels to prevent or minimize various side-channel attacks.

Nowadays, the computing systems of many consumers include separate secure cryptographic processor called Trusted Platform Module (TPM). Even though the whole computing platform is insecure, a part of it is guaranteed to be secure. TPM can encrypt and save important information such as software keys in shielded locations [10] which can only be accessed with special permission. Any security violation due to a software intrusion is reported and blocked 
by generating hardware interrupts. This can improve security and trust of computing system by thwarting several software related vulnerabilities.

Because of existing IP reuse-based approach, the IPs used in modern IC designs are susceptible to copyright infringements [11]. In addition, counterfeit chips may be produced by a malicious manufacturer abroad from illegally obtained IPs e.g. by espionage, theft, reverse engineering etc. to sell them in very low price [12]. Various authentication based or watermarking schemes have been proposed for IP protection [11,13-17] at different abstraction levels. Active $[12,18]$ and passive IC metering techniques allow IP providers to keep track of the chips produced from sold IPs. The active metering method uniquely locks each manufactured chip which needs specific key provided by IP owner [18]. Current passive IC metering techniques create IC fingerprint using gate delays, leakage power, switching power etc. In Physical Unclonable Function (PUF) based IC authentication technique, unique physical randomness like process variation is used which creates unique challenge-response pair for each fabricated chip [19-22].

Reverse engineering of an IC can be used to reconstruct the circuit, and extract the complete design and functionality. It involves many steps including x-ray of the chip, acid etching, reactive-ion etching, scanning electron microscope imaging, layout extraction etc. It is commonly used to benchmark the products, identify the patent infringements, trace the cause of chip failure and replace the non-functional out-of-date chips of long-lasting products such as military devices, ships, nuclear reactors etc. [23, 24]. However, it can be used for malicious purposes too. For example, the extracted design secret of a military 
IC can be used to manufacture its identical chips to be used in several deadly weapons. By understanding the reverse-engineered design, intelligent Trojans can also be inserted by altering the masks at fabrication stage. Various anti-tamper techniques obfuscate the hardware design at Register-Transfer Level (RTL) to make a fabricated chip extremely hard to reverse-engineer $[25,26]$. In $[25,26]$, a small Finite State Machine (FSM) is added to modify existing state-transition function of the design. The input of this additional FSM is a subset of primary inputs. There are two modes of operation: (1) obfuscation mode, and (2) normal mode. The FSM starts from reset state and makes transition to different states in obfuscation mode depending upon different input sequences. It can enter into normal mode only after the application of a pre-defined initialization key sequence provided by IP owner.

Since Trojans can be inserted at any stage of the design flow by an attacker, necessary measures must be taken to capture them at different design stages. If the Trojan is inserted in a pre-silicon stage, formal verification methods such as Sequential Equivalence Checking (SEC) and test generation techniques can be extremely useful to detect them. Since static timing and power analyses are performed in the Trojan-infested circuit, they are not be useful for Trojan detection in post-silicon stage. If malicious insertions are made during or after fabrication, conventional post-silicon testing methods for manufacturing defects including tests for stuck-at faults, path-delay faults, $I_{D D Q}$ faults etc. won't be able to detect them. Automatic Test Pattern Generation (ATPG) tries to generate a test vector that excites a fault and propagate its effect to the primary output. But, the faults pertinent to the Trojan inserted in fabrication stage will not be exercised since ATPG vectors are generated for the 
original Trojan-free circuit. Therefore, even though ATPG generates 100\% fault coverage, Trojans will escape during post-manufacturing testing.

\subsection{Contribution}

In this thesis, we concentrate on ensuring trust in hardware designs by the detection of any malicious insertion in third-party IP modules. Existing SEC techniques try to prove if the design under verification is functionally equivalent to the reference design. But this equivalence checking is itself challenging and a very hard problem. They assume that the ideal reference golden model is available and the design stage is within trust boundary. Because of the trust issues with the use of IPs acquired from third party vendors, additional efforts are needed to guarantee the trust of such IPs. We focus on the use of modified IC along with test generation method to identify malicious output behavior of malicious designs because of embedded Trojans. We first logic simulate the suspect circuit using a large number

of random vectors to identify suspicious signal candidates and learn the potential invariants among the signals within the suspect circuit and also with the signals of reference spec circuit. As soon as we get the list of proven invariants, we perform SEC to check if the true invariants are enough to prove the functional equivalence of suspect and spec circuits [27].

Next, we apply two-step approach to conclude about the suspicious signals. In the first step, we make sure that the suspicious signal under consideration in the suspect circuit is activated and propagated to a primary output with an ATPG. And in the second step, 
counterexample guided equivalence checking is employed to check the output behavior of two circuits. Experimental results for a set of hard-to-verify designs show that our method can either ensure that the suspect design is free from the functional effect of any malicious change(s) or return a small group of most likely malicious signals, all in a short amount of time.

\subsection{Organization}

The rest of the thesis is organized as follows.

Chapter 2 describes Trojan characteristics and classification. It discusses the previous works related to Trojan detection at pre-silicon and post-silicon stages. It also explains different terminologies and basic concepts related to miter construction, model checking, equivalence checking, suspect signals generation, proving invariants and their use in sequential equivalence checking.

Chapter 3 explains our proposed method to detect malicious inclusions in third-party IPs. It discusses invariant based test generation and counterexample guided equivalence methods in detail.

Chapter 4 describes the modifications in the proposed technique to generate more effective counterexample.

Finally, Chapter 5 concludes the thesis and gives some direction about the future work. 


\section{Chapter 2}

\section{Background}

\subsection{Trojan Characteristics and Classification}

A hardware Trojan is an intentional, tiny and stealthy modification in the circuit logic at any abstraction level. It is dormant for the most part of the device's operation. Once it is triggered, it shows its effect to the payload and the effect may propagate to the output depending upon other circuit parameters. A hardware Trojan can be combinational or sequential [28-30]. A combinational Trojan consists of only combinational logic gate(s) whereas a sequential Trojan is a finite state machine (FSM) that gets triggered by a sequence of internal signal conditions. Fig. 2.1 shows an example of multiplexer as a combinational Trojan $[31,32]$ which bypasses a portion of circuit logic if triggered. The Trojan gets triggered if we get a rare logic ' 0 ' at node $e$. 


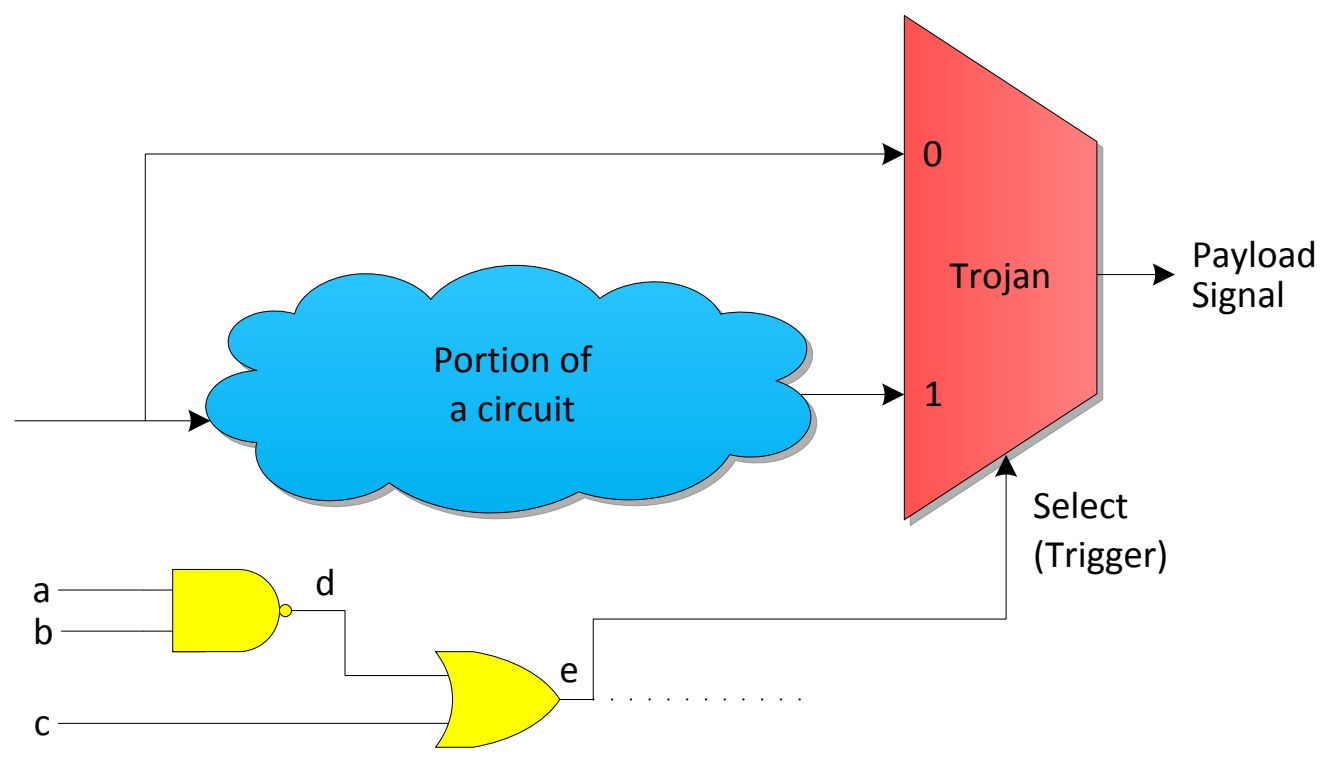

Figure 2.1: Example of a simple Trojan.

Source: R. Karri, J. Rajendran, K. Rosenfeld, and M. Tehranipoor, Trustworthy hardware: Identifying and classifying hardware Trojans, IEEE Computer, Vol. 43, No. 10, Oct. 2010, pp. 39-46. Used under fair use, 2012.

Researchers have proposed different ways of classifying Hardware Trojans [31-35]. In [33], the authors categorize them into explicit payload Trojan and implicit payload Trojan according to behavior of Trojan payload. The explicit payload Trojan consists of a circuit node as payload signal and the trigger effect gets propagated through payload to the primary output. The trigger part of the implicit payload Trojan is similar to explicit payload Trojan but the payload may leak secret information by emitting electromagnetic signals or may destroy the entire IC. The authors of [34] classify Trojans on the basis of their trigger and payload mechanisms.Both trigger and payload may be analog or digital. The trigger can be delayed by asynchronous or $k$-bit synchronous counter. A digital payload may alter control, status 
or data line whereas analog payload may drain battery faster, cause bridging faults etc. A detailed classification based on physical, activation and action characteristics is illustrated in [35]. The most comprehensive Trojan taxonomy is presented in [31,32] according to insertion phase, abstraction level, activation mechanism, effects and location as shown in Fig. 2.2.

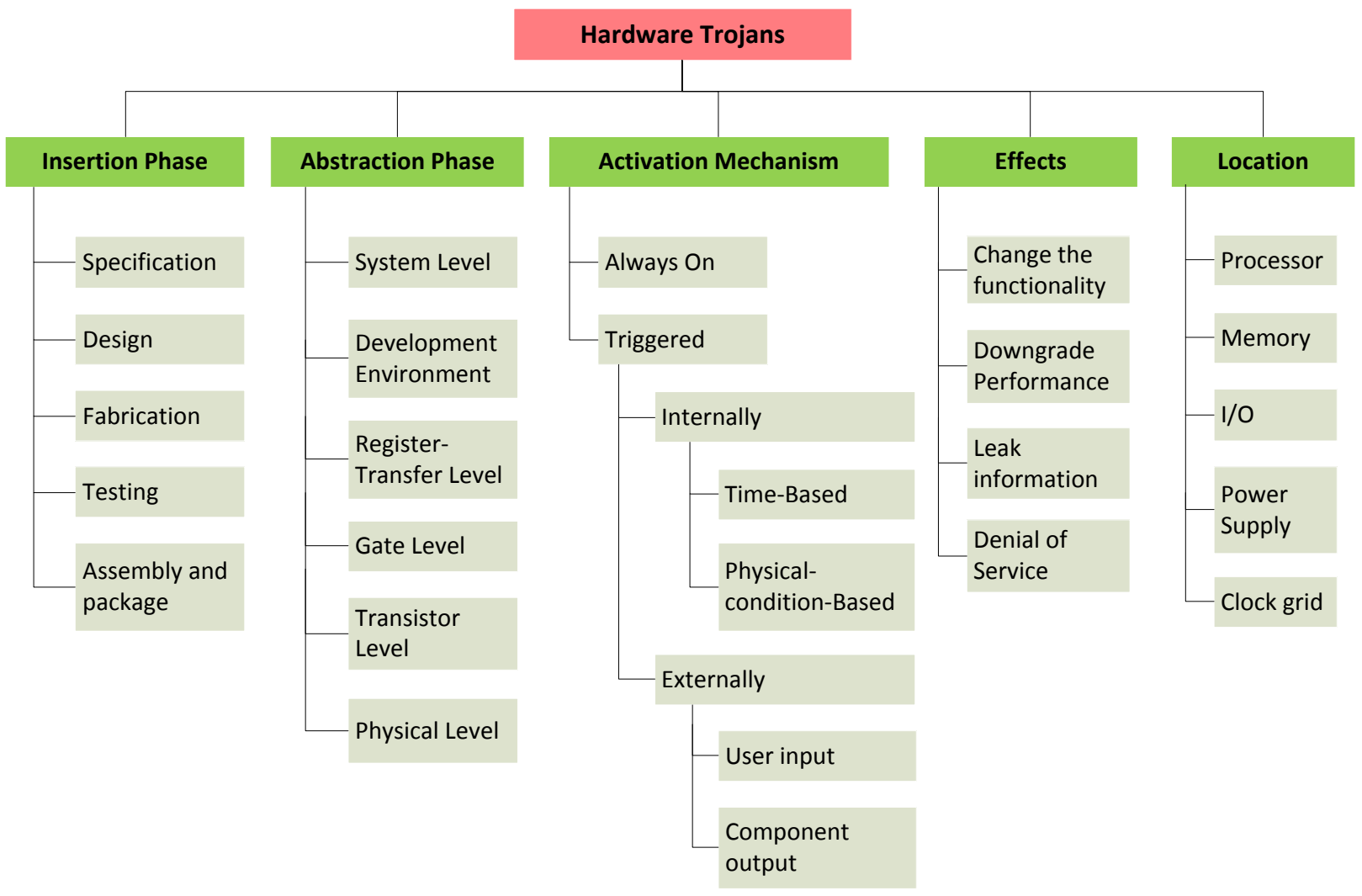

Figure 2.2: Trojan Classification.

Source: R. Karri, J. Rajendran, K. Rosenfeld, and M. Tehranipoor, Trustworthy hardware: Identifying and classifying hardware Trojans, IEEE Computer, Vol. 43, No. 10, Oct. 2010, pp. 39-46. Used under fair use, 2012. 


\section{Insertion Phase}

- Specification: Modification of functional specifications or other constraints like timing, area and power requirements in the design.

- Design: Alterations can be made in third-party IPs, Computer-Aided Design (CAD) tools [36], standard cells or models.

- Fabrication: Masks can be altered.

- Testing: Testing phase is the stage to detect any manufacturing defects and Trojans in the fabricated chip. Modifications are possible in this phase by manipulating the test vectors.

- Assembly and Package: Wires in PCB too close or unshielded to create electromagnetic coupling.

\section{Abstraction Level}

- System Level: Insertions can be made in complex hardware modules, and interconnections and communications among multiple modules.

- Development Environment: Modifications in automated scripts, IP libraries, CAD tools [36] etc.

- Register-Transfer Level: Manipulation of any underlying state machines, adding extra logic, erasing a portion of logic or modifying the existing logic [37].

- Structural Level: Intelligent modification, addition and deletion of logic gates. 
- Transistor Level: Change of different transistor parameters e.g. timing, power characteristics etc.

- Physical Level: Change in layout of gates, metal layers, interconnects etc.

\section{Trigger Mechanism}

- Always activated: Trojan is always active.

- Internally triggered: The internal event to cause Trojan activation can be timebased (e.g., counter to count for a specific duration before trigger) or physical-conditionbased (e.g., trigger mechanism waits till specific temperature).

- Externally triggered: The external trigger mechanism is based on the user's input (e.g., keyboard input) or component output (e.g., specific output from another module connected to the input of the Trojan-infested module).

\section{Activation Effects}

- Change the functionality: Toggles the output; wrong results.

- Downgrade performance: Addition of delay; timing violations; power delay; gate delay etc.

- Leak secret key information: Through wireless channel [38] \& side-channel power.

- Denial of service: Temporary (e.g. generates interrupt) or permanent (e.g. destroys the chip). 


\section{Trojan Location}

- Processor: Backdoor insertion can be exploited by software during operation [2].

- Memory: Manipulates the stored information in memory; violates memory access.

- I/O peripherals: Records keystrokes, leak secret information through RS-232 cable.

- Clock grid: Degrades performance by inserting clock glitches, changing frequency etc. [31].

\subsection{Previous Works}

Researchers have proposed many non-invasive techniques to detect embedded Trojans in the fabricated chips [29,30,33,39-43] assuming that Trojans are inserted during manufacturing. In $[39,40]$, the authors use side channel power analysis to obtain the power signature that differentiates malicious and genuine ICs. In [29], the authors present a method of maximizing toggle in the targeted circuit regions. A sustained vector methodology is proposed to minimize circuit activities and identify extraneous toggles caused by Trojan circuit in [30]. In addition, the authors of [33] discuss the method to measure the path delay at the output ports for a set of input vectors and identify the additional delay introduced by Trojans in the path where it resides. A high-precision path delay measurement test structure having extremely low overhead is proposed to detect path delays introduced by Hardware Trojans in [41]. The authors of [42] show that strong detectability of Hardware Trojans is signifi- 
cantly increased by using statistical analysis in delay-based techniques. In [43], the authors maximize the probability of Trojan detection by generating a minimal set of test vectors that causes excitation of a node in the circuit for a specified number of times. The authors of [44] use gate level characterization technique using power measurements and errors minimization by formulation of linear equations to detect malicious insertions.

Apart from various post-silicon Trojan detection methodologies, there is a need of efficient and cost-effective mechanism to reliably detect and/or prevent the Trojan effects at earlier IC design stages. Trojans inserted at a pre-silicon stage, if not detected before fabrication, would make their presence in all manufactured ICs. A few recent research targeting Trojans inserted at pre-silicon stages are based on formal verification, code coverage analysis and ATPG methods. The authors of [45] mention how FSM can be exploited to insert stealthy Hardware Trojans by a designer making them extremely hard to diagnose. In [46], a Design for Trojan Test (DFTT) methodology is developed in which user-generated RTL code is converted into DFTT compliant code. Sensitive paths susceptible to Trojan insertions are also identified in the design. Finally, probe cells are inserted into these paths and test vectors are generated to detect malicious insertions either during design or fabrication. In [47], the authors propose BlueChip (a defensive strategy having both design-time and runtime components) that identifies and removes unused suspicious circuitry inserted during design, and uses software at runtime to emulate hardware instructions related to the removed circuitry. In $[37,48]$, IP acquisition and delivery protocols are proposed in which IP vendors provide not only Hardware Description Language (HDL) codes but also the proof of security 
properties. In [49], the author presents a technique to use untrusted CAD tools to synthesize trustable IC designs.

Different pre-silicon trust verification strategies involving formal verification, code coverage analysis and techniques to reduce suspicious signals by redundancy removal, equivalence analysis and sequential ATPG are studied in [50]. The authors of [51] propose a methodology to compare the functionality of two untrusted similar third-party IPs. The number of inputs is made identical in both IPs by encapsulation of wrapper in one of them. The two circuits are then unrolled to multiple timeframes to remove the internal states and make the outputs function of only present and past inputs. An approach which involves N-detect full-scan ATPG method, along with Suspect Signal Guided Sequential Equivalence Checking (SSG-SEC) to identify malicious signals corresponding to hard-to-detect faults is presented in [52]. A region isolation method is employed to locate the Trojan signals in the design. During SSG-SEC, a triple miter is constructed using two copies of suspicious circuit and a copy of the spec circuit.

\subsection{Preliminaries}

\subsubsection{Suspicious Signals}

Suspicious signals [52] are those signals which toggle little (remain dormant) during the circuit operation, have very few activation and propagation sequences, or those which flip 
a lot but their activation effects do not seem to affect the output. A suspicious signal is the perfect signal in the circuit to be exploited by the Trojan because they cannot be easily detected via simulation. The rest of the signals in the circuit are considered to be non-suspicious.

\subsubsection{Path Sensitization}

A node is sensitive to its input node if the logic value of that node gets toggled by complementing the input node. Consider an AND gate with three inputs $a, b$ and $c$ and output $d$. If one of the input of the gate is assigned ' 0 ' say $b=0$, then output of the gate is $d=0$. Here, logic ' 0 ' is the controlling value for AND gate. However, to make $d$ sensitive to $b$, we need to make rest of the inputs $a$ and $c$ to be non-controlling, i.e. logic ' 1 ' for AND gate. Therefore, the path in a circuit can be sensitized if each of its nodes is sensitive to the predecessor nodes along the same path. If the path is being sensitized for fault effect propagation, the origin of this path is the fault site.

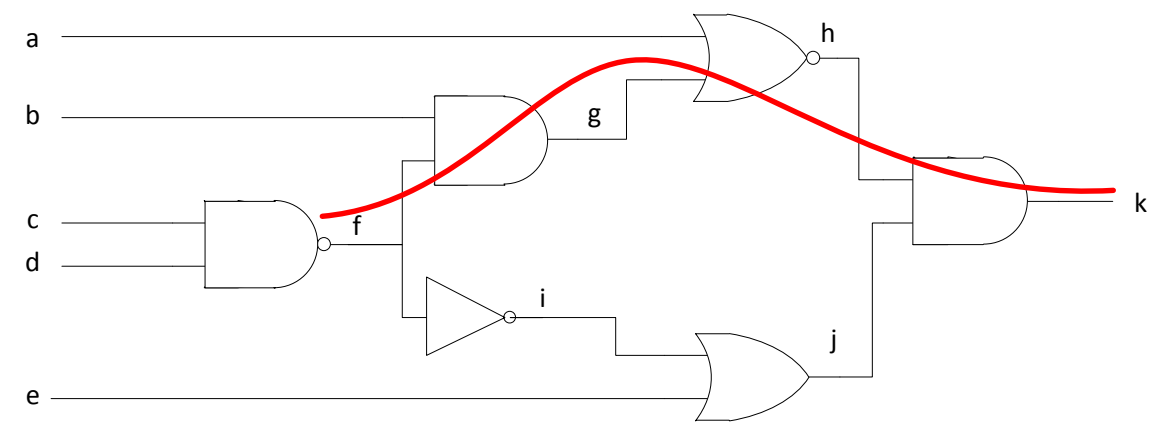

Figure 2.3: Example for Path Sensitization 
Fig. 2.3 shows a simple combinational circuit where we want to sensitize a path starting from node $f$ to the primary output $k$. The path $f-g-h-k$ can be sensitized if the off-path inputs to the gates $g, h$ and $k$ are non-controlling. This can be done by assigning the input values $a=0, b=1$ and $e=1$.

\subsubsection{Static Logic Implication}

Static logic implication or static learning [53] is the effect of a node assignment in the rest of the circuit. The assignment of a logic value ' 0 'or ' 1 ' at a particular node may imply a logic value at another node in the circuit. For example, if a node $A$ of logic value $v, v \in(0,1)$ implies node $B$ of value $w, w \in(0,1)$ after $k$ timeframes, then we can represent the implication as $(A, v) \rightarrow(B, w, k)$. By contrapositive law, the implication of $(B, \neg w)$ would be $(B, \neg w) \rightarrow(A, \neg v,-k)$. On the other hand, if $(B, w) \rightarrow(C, x, l)$ then, according to law of transitivity, the newly formed implications are $(A, v) \rightarrow(C, x, k+l)$ and $(C, \neg x) \rightarrow$ $(A, \neg v,-(k+l))$ (contrapositive).

Static learning methods have been extremely useful in various applications like ATPG, logic optimization, untestable fault identification, design verification etc. There are mainly three types of static implications: direct, indirect and extended backward implications. Various methods have been proposed to compute two-node and multi-node implications in combinational as well as sequential circuits [53-55]. The authors of [53] use an iterative approach to compute static implications in combinational circuits. In [54], the authors use implica- 
tion graph to efficiently store the learned implications in sequential circuits. Moreover, a technique to generate multi-node implications in combinational circuits is explained in [55].

\section{Direct Implication}

Direct implication of a node assignment is the implication associated with the inputs and outputs of a gate. There are two types of direct implications: direct forward and direct backward implications. Direct forward implications are computed according to the controlling value of the gate under consideration whereas direct backward implications are contrapositive of direct forward implications. Consider nodes $X$ and $Y$ be two inputs of AND gate whose output is node $Z$. Since logic ' 0 ' is the controlling value for AND gate, if any of the inputs of AND gate is logic ' 0 ', the output of AND gate is also logic ' 0 ' irrespective of the logic value(s) of rest of the inputs. Therefore, we can derive the direct forward implication, $(X, 0) \rightarrow(Z, 0,0)$ and $(Y, 0) \rightarrow(Z, 0,0)$. Direct backward implication is the contrapositive of direct forward implication. Therefore, in previous example, the direct backward implications would be $(Z, 1) \rightarrow(X, 1,0)$ and $(Z, 1) \rightarrow(Y, 1,0)$. For a D Flip-flop of input node $A$ and output node $B$, direct forward implications can be derived as $(A, 0) \rightarrow(B, 0,1)$ and $(A, 1) \rightarrow(B, 1,1)$, and the direct backward implications would be $(B, 0) \rightarrow(A, 0,-1)$ and $(B, 1) \rightarrow(A, 1,-1)$.

Table 2.1 shows different gate types, their controlling values, direct forward implications and direct backward implications associated with them. We assume inputs of a gate as $a$ and $b$ and output as $c$. 
Table 2.1: Table Showing Direct Forward And Backward Implications For Different Gates

\begin{tabular}{|c|c|c|c|c|}
\hline $\begin{array}{c}\text { Gate } \\
\text { type }\end{array}$ & $\begin{array}{c}\text { Controlling } \\
\text { Value }\end{array}$ & $\begin{array}{c}\text { Non-Controlling } \\
\text { Value }\end{array}$ & $\begin{array}{c}\text { Direct Forward } \\
\text { implication }\end{array}$ & $\begin{array}{c}\text { Direct Backward } \\
\text { Implication }\end{array}$ \\
\hline AND & 0 & 1 & $\begin{array}{c}(a, 0) \rightarrow(c, 0,0) \\
(b, 0) \rightarrow(c, 0,0)\end{array}$ & $\begin{array}{c}(c, 1) \rightarrow(a, 1,0) \\
(c, 1) \rightarrow(b, 1,0)\end{array}$ \\
\hline OR & 1 & 0 & $(a, 1) \rightarrow(c, 1,0)$ & $(c, 0) \rightarrow(a, 0,0)$ \\
& & & $(b, 1) \rightarrow(c, 1,0)$ & $(c, 0) \rightarrow(b, 0,0)$ \\
\hline NAND & 0 & 1 & $(a, 0) \rightarrow(c, 1,0)$ & $(c, 0) \rightarrow(a, 1,0)$ \\
& 1 & & $(b, 0) \rightarrow(c, 1,0)$ & $(c, 0) \rightarrow(b, 1,0)$ \\
\hline NOR & 1 & 0 & $(b, 1) \rightarrow(c, 0,0)$ & $(c, 1) \rightarrow(a, 0,0)$ \\
& & & $(c, 0,0)$ & $(c, 1) \rightarrow(b, 0,0)$ \\
\hline
\end{tabular}

\section{Indirect Implication}

Indirect implication is the implication computed by logic simulation of the circuit assigning the transitive closure of the node of interest. Before logic simulation, we place the existing node assignments of transitive closure and assign rest of the nodes as unknown in the circuit. After logic simulation, additional gates may be evaluated to known value either logic ' 0 ' or '1'. The newly learned node assignments can be added to the implication graph as indirect implication.

Fig. 2.4 illustrates a simple circuit which consists of four inputs namely $a, b, c$ and $d$, and two outputs $g$ and $h$. The direct implications of $(b, 0)$ are $(e, 1,0)$ and $(f, 1,0)$. Similarly, 


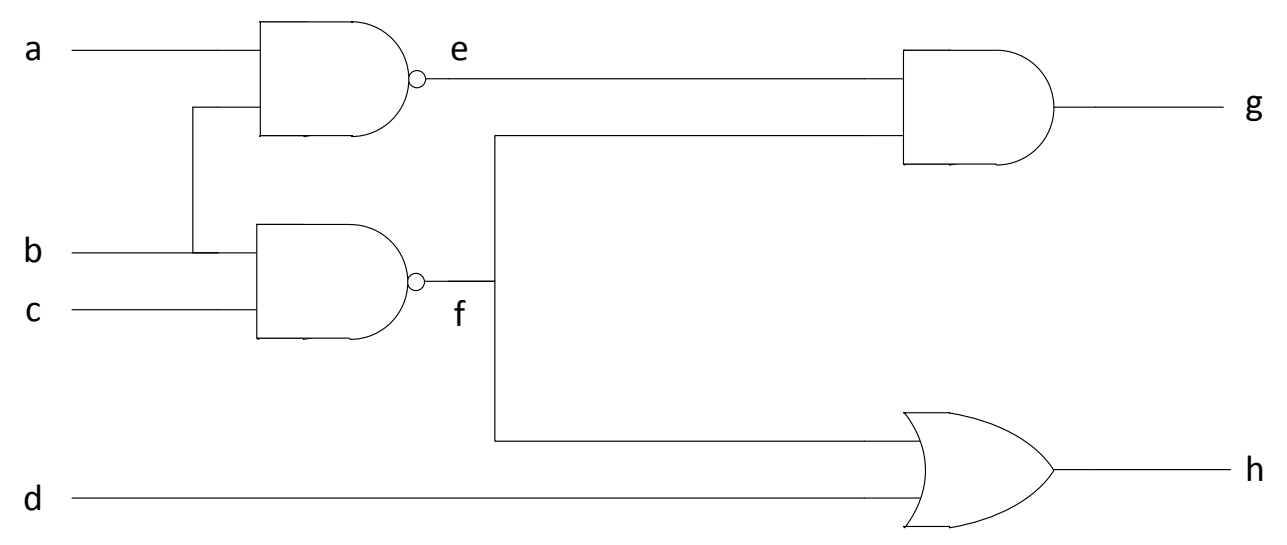

Figure 2.4: Example of Direct and Indirect Implications

direct implication of $(f, 1)$ is $(h, 1,0)$. By law of transitivity, the new implication learned is $(b, 0) \rightarrow(h, 1,0)$. Now the transitive closure of $(b, 0)$ is $(b, 0,0),(e, 1,0),(f, 1,0),(h, 1,0)$. To find the indirect implication of $(b, 0)$, all the implication values in the transitive closure are assigned in the circuit and rest of the gate values are kept unknown. After logic simulation, gate $g$ is evaluated to logic ' 1 ' and thus the assignment $(g, 1,0)$ is learned which is considered as indirect implication of $(b, 0)$.

Fig. 2.5 shows the implication graph constructed for the node assignment $(b, 0)$ for circuit in Fig. 2.4. The directed edge with solid line represents the direct implication and the dotted line represents the indirect implication. The weight in the directed edge signifies the implication distance. For the combinational circuit, all the edge weights are zero. 


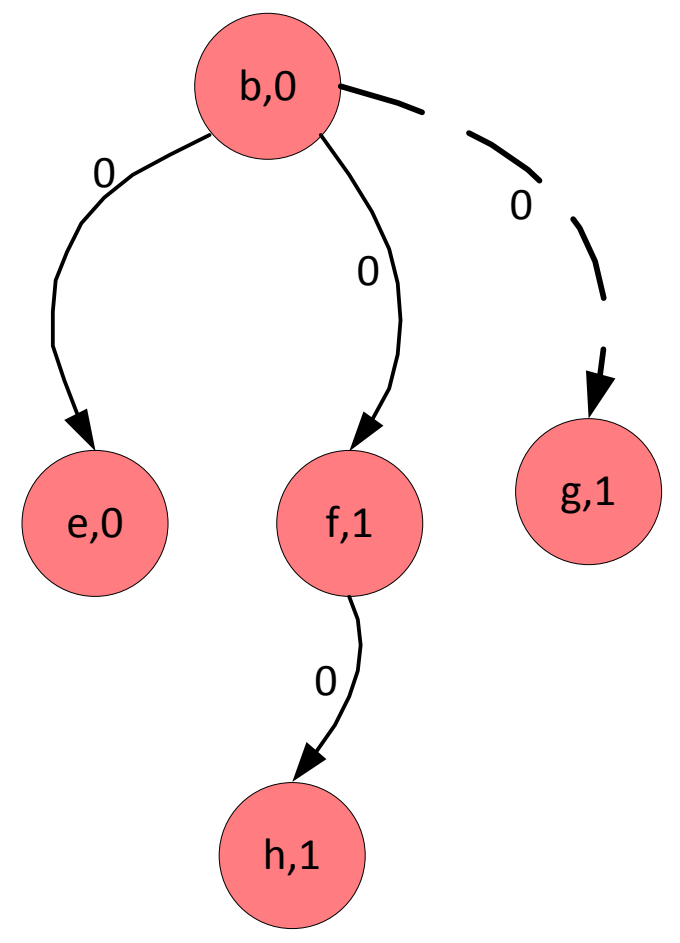

Figure 2.5: Implication Graph Example

\subsubsection{Boolean Satisfiability (SAT) and Conjunctive Normal Form}

Binary Decision Diagrams (BDDs) and Boolean Satisfiability are the two extensively used methods in formal verification. However, BDDs are less popular because of limited scalability due to memory explosion problems. A Satisfiability (SAT) problem is converted into propositional formula $f$ in Conjunctive Normal Form $(\mathrm{CNF})$ that can be interpreted by a SAT solver. The computational complexity of a general SAT problem has been found to be NP-complete [56]. However, a number of very efficient SAT solvers have been proposed which can successfully handle big industrial designs viz. MiniSAT [57], Zchaff [58], GRASP [59] and so on. All of them are based on search tree and are derivatives of DPLL algorithm [60]. 
Table 2.2: Table Showing CNF Formulation For Different Gates

\begin{tabular}{|c|c|c|c|}
\hline Gate type & $\mathrm{I} / \mathrm{O}$ relation & Implication & CNF \\
\hline NOT & $\mathrm{c}=\bar{a}$ & $\begin{array}{l}c \rightarrow \bar{a} \\
\bar{c} \rightarrow \bar{a}\end{array}$ & $(\neg c \vee \neg a) \wedge(c \vee a)$ \\
\hline BUFFER & $\mathrm{c}=\mathrm{a}$ & $\begin{array}{l}c \rightarrow a \\
\bar{c} \rightarrow \bar{a}\end{array}$ & $(\neg c \vee a) \wedge(c \vee \neg a)$ \\
\hline AND & $\mathrm{c}=a . b$ & $\begin{array}{l}c \rightarrow a * b \\
\bar{c} \rightarrow \bar{a}+\bar{b}\end{array}$ & $\begin{array}{l}(\neg c \vee a) \wedge(\neg c \vee b) \\
\wedge(c \vee \neg a \vee \neg b)\end{array}$ \\
\hline OR & $\mathrm{c}=a+b$ & $\begin{array}{l}c \rightarrow a+b \\
\bar{c} \rightarrow \bar{a} * \bar{b}\end{array}$ & $\begin{array}{l}(c \vee \neg a) \wedge(c \vee \neg b) \\
\wedge(\neg c \vee a \vee b)\end{array}$ \\
\hline NAND & $\mathrm{c}=\overline{a \cdot b}$ & $\begin{array}{l}c \rightarrow \bar{a}+\bar{b} \\
\bar{c} \rightarrow a * b\end{array}$ & $\begin{array}{l}(c \vee a) \wedge(c \vee b) \\
\wedge(\neg c \vee \neg a \vee \neg b)\end{array}$ \\
\hline NOR & $\mathrm{c}=\overline{a+b}$ & $\begin{array}{l}c \rightarrow \bar{a} * \bar{b} \\
\bar{c} \rightarrow a+b\end{array}$ & $\begin{array}{l}(\neg c \vee \neg a) \wedge(\neg c \vee \neg b) \\
\wedge(c \vee a \vee b)\end{array}$ \\
\hline $\mathrm{XOR}$ & $\mathrm{c}=a \bar{b}+\bar{a} b$ & $\begin{array}{l}c \rightarrow a * \bar{b}+\bar{a} * b \\
\bar{c} \rightarrow a * b+\bar{a} * \bar{b}\end{array}$ & $\begin{array}{l}(\neg c \vee \neg a \vee \neg b) \wedge(\neg c \vee a \vee b) \\
\wedge(c \vee \neg a \vee b) \wedge(c \vee a \vee \neg b)\end{array}$ \\
\hline XNOR & $\mathrm{c}=a b+\bar{a} \bar{b}$ & $\begin{array}{l}c \rightarrow a * b+\bar{a} * \bar{b} \\
\bar{c} \rightarrow a * \bar{b}+\bar{a} * b\end{array}$ & $\begin{array}{l}(c \vee \neg a \vee \neg b) \wedge(c \vee a \vee b) \\
\wedge(\neg c \vee \neg a \vee b) \wedge(\neg c \vee a \vee \neg b)\end{array}$ \\
\hline
\end{tabular}

A Conjunctive Normal Form $(\mathrm{CNF})$ is defined as a conjunction of clauses. A clause is a disjunction of literals. A literal is the positive or negative polarity of a boolean variable. A SAT solver tries to find if there exists any assignment that makes the propositional formula $f$ true. If there does not exist any solution, the SAT problem will be unsatisfiable. A SAT 
solver will return satisfiable with a counterexample that acts as a witness to make $f$ true.

To convert the whole circuit into CNF, we need to consider the relationships between inputs and outputs of each gate in the circuit. For example, for a two-input AND gate with two inputs $a$ and $b$ and output $c$, the relation $c=a * b$ or $c \rightarrow a * b, \neg c \rightarrow \neg(a * b)$ can be represented in propositional formula as $(\neg c \vee a) \wedge(\neg c \vee b) \wedge(c \vee \neg a \vee \neg b)$. Similarly, for a buffer with input $p$ and output $q$, CNF will be $(q \vee \neg p) \wedge(\neg q \vee p)$.

Table 2.2 illustrates different gate types, their I/O relation, I/O relation in terms of Implication and CNF representation. The gates are assumed to have two inputs $a$ and $b$ except inverter and buffer. For inverters and buffers, only input $a$ is present. For all gates the output is $c$.

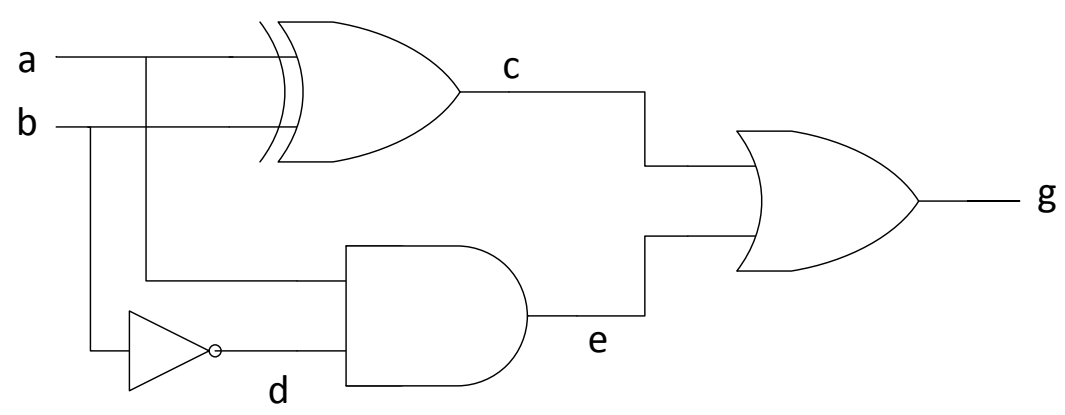

Figure 2.6: A simple combinational circuit for CNF conversion

Consider a simple circuit as shown in Fig. 2.6. The variables of the SAT problem can be represented by the gate IDs in the circuit. The propositional formula $f$ for the circuit using Table 2.2 is given by, 


$$
\begin{aligned}
f= & (\neg c \vee a \vee b) \wedge(\neg c \vee \neg a \vee \neg b) \wedge(c \vee \neg a \vee b) \wedge(c \vee a \vee \neg b) \wedge \\
& (d \vee b) \wedge(\neg d \vee \neg b) \wedge(e \vee \neg a \vee \neg d) \wedge(\neg e \vee a) \wedge(\neg e \vee d) \wedge \\
& (\neg g \vee c \vee e) \wedge(g \vee \neg c) \wedge(g \vee \neg e)
\end{aligned}
$$

\subsubsection{Model Checking}

Model checking is the technique to verify a set of properties in the design. The properties are represented in temporal logic and the system is modeled as a Kripke structure or state transition graph. It traverses the transition graph and checks for any state that violates specified property. Instead of traversing each and every reachable state space, symbolic model checking models the transition graph into BDDs or satisfiability problem. Since BDDs are susceptible to memory explosion, modern symbolic model checking methods use SAT solver as underlying engine to handle large industrial designs instead of BDDs.

Symbolic model checking can be categorized as:

(I) Bounded Model Checking (BMC)

(II) Unbounded Model Checking (UMC)

\section{Bounded Model Checking (BMC)}

Given a property $p$, BMC [61] first unrolls the transition relation $k$ times and searches for a sequence of length $k$ from a reachable starting state that violates the property in the unrolled 
bound. If $\neg p$ can be reached at any state from the initial state, it returns a counterexample; otherwise we increment $k$ until we reach a predetermined threshold. However, even if SAT solver is unable to find the counterexample, BMC cannot guarantee the truthfulness of the property because the property may be violated in a reachable state which is outside the unroll threshold.

Induction based BMC [62] involves inductive analysis to prove the validity of the property. The first step is the base case, in which we verify that property $p$ holds in a known valid reachable state $S_{0}$. In the induction step, we assume that the property $p$ holds for any reachable state $S_{k}$ and we check if $p$ can be falsified in the next state $S_{k+1}$. If the SAT solver returns a counterexample, the states $S_{k}$ and $S_{k+1}$ obtained may not be the true reachable states since there is no way to constrain all the unreachable states that can violate $p$. But, if there exists no counterexample, the property holds true for all the states reachable from $S_{0}$. Similarly, to prove the property $p$ by strong induction, in the base case we verify that $p$ is valid for all the transition sequences of length $k$ starting from the state $S_{0}$. In the induction case, we assume that the property is valid for any $k$ transition sequence of reachable states and check if the property gets violated in the next reachable state.

\section{Unbounded Model Checking (UMC)}

Unbounded model checking [63] guarantees the truthfulness of the property if declared so, since it computes all reachable states from an initial state. Consider a system having $s$ state variables and $i$ inputs. Let $S_{0}(s)$ be the set of initial states, $\phi$ be the property under 
verification and $T\left(s, i, s^{\prime}\right)$ be the transition relation. Unbounded model checking computes image (immediate successor) of state $S(s)$ by operator given by,

$$
\operatorname{Img}(S(s))=\exists s . T\left(s, i, s^{\prime}\right) \wedge S(s)
$$

The next state sets are computed by applying above operator until we reach fix-point that gives all the reachable state space starting from $S_{0}(s)$. Then we check if any of the reachable states falsifies the property $\phi$. If there is any violation, a counterexample is obtained starting from the state $S_{0}$. If there is no counterexample, property $\phi$ is guaranteed to be true. Unbounded model checking for large industrial design is extremely difficult because there are chances of memory and temporal explosion.

\subsubsection{Equivalence Checking and Miter Circuit}

Equivalence Checking is a special case of model checking which is used to ensure if the two circuits under verification (CUVs) are functionally equivalent. The circuits may be without any reset mechanism and may be at any state (reachable or unreachable) when powered on. But they may reach a known reachable state by the application of initialization sequence. Simulation-based equivalence checking methods work only for small circuits, as they cannot cover all allowable input combinations for all reachable states in large designs. Formal equivalence checking treats equivalence checking as a formal property and checks if there exists any state that violates this property.

Given two finite state machines (FSMs) to prove equivalence, a product machine can be cre- 
ated and starting from a known initial state, all the reachable state space of product machine can be computed. For any computed reachable state, the two FSMs should give identical outputs to prove them equivalent. But computing all reachable state space in large VLSI design is extremely difficult. Therefore, a number of induction based sequential equivalence checking algorithms have been proposed $[27,64-66]$ in recent years that exploit structural similarities and signal relations to prove equivalence. However, sequential equivalence checking (SEC) is still a hard problem because the two circuits under verification may have very little resemblance of state variables and internal signals.

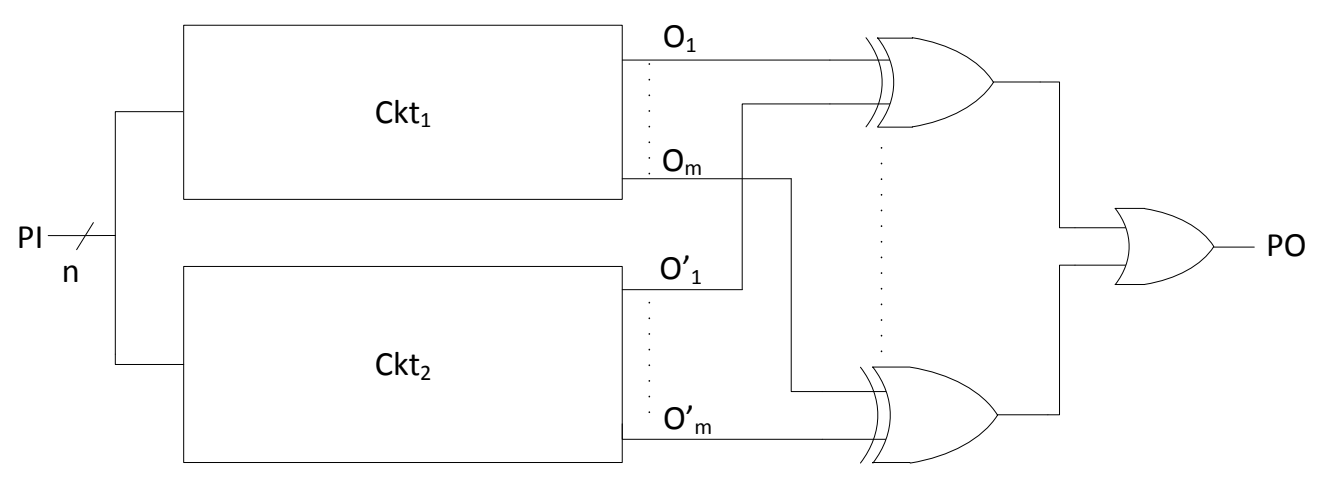

Figure 2.7: Miter Circuit

During SAT-based formal equivalence checking, a miter circuit is created from two CUVs by connecting their corresponding Primary Input (PI) pairs together, XORing the corresponding Primary Output (PO) pairs (and ORing these XOR outputs if multiple PO pairs are present). Fig. 2.7 shows a miter circuit constructed from two circuits $C k t_{1}$ and $C k t_{2}$ having $n$ inputs and $m$ outputs. The property to prove functional equivalence $p$ can be represented by the final PO to be constant logic ' 0 '. 


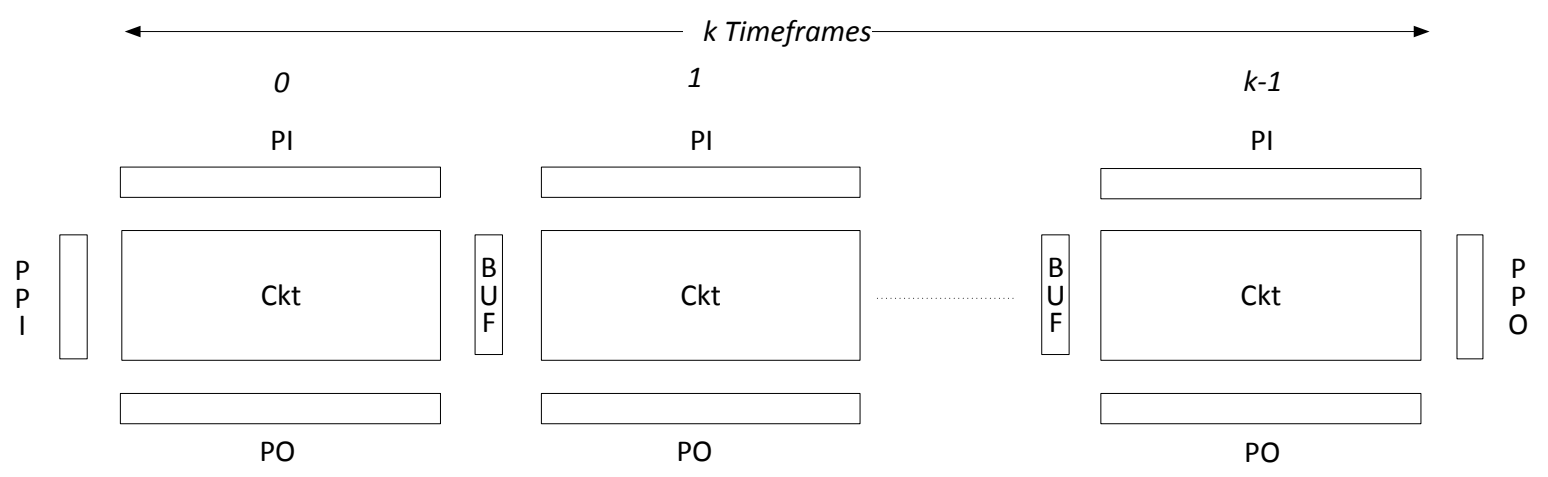

Figure 2.8: Unrolled Circuit

For combinational circuit, only one copy of the miter circuit is converted into CNF and fed to the SAT solver. We seek if there exists an assignment that distinguishes the two circuits under verification (CUVs) by forcing the final OR gate to logic ' 1 ' and adding it as constraint to the SAT solver. If the SAT solver gives a satisfying assignment, the input test set acts as witness to distinguish two combinational circuits and thus the circuits can be declared as not equivalent, whereas if it returns unsatisfiable, they are concluded as equivalent.

Since a sequential circuit consists of state variables, only one instance of the sequential miter circuit is not enough. The miter needs to be unrolled for $k$ timeframes within the unroll limit to convert it into the combinational circuit. While unrolling, all the circuit elements are copied $k$ times representing each of the timeframe instances as shown in Fig. 2.8. Flipflops at the left-most timeframe are treated as pseudo-primary inputs (PPIs) and those on the other timeframes are converted into buffers between the adjacent timeframes. The final state elements at the right-most timeframe are considered as pseudo-primary outputs (PPOs). The constraint for equivalence property is a clause obtained by ORing final OR 
gates of all unrolled timeframes. Pseudo-Primary Input (PPI) represents the starting state of the miter circuit which needs to be reachable. To do so, we need to add extra constraints that can block unreachable states at PPI. If a SAT solver gives a valid satisfying solution for any of the final OR gates to be logic ' 1 ', it signifies that there exists at least one vector that can distinguish the two CUVs.

\subsubsection{Invariants}

Invariants are the relationships among signals in the circuit. Static logic implications (discussed in subsection 2.3.3) are those invariants which hold true for all the states (both reachable and unreachable states) of the design. They are not powerful in pruning the search space during SAT solving since they cannot be used to separate the unreachable states from the reachable ones. Inductive invariants, on the other hand, are those relations that definitely hold true in the reachable state space of the design but may not be true in the unreachable ones. The inductive invariant, if applied in SAT solving, will block the unreachable states which violate it.

For example, an invariant $(a \vee b \vee c)$ indicates that in every legal state of the circuit, at least one of $a, b$ and $c$ must be true. However, some unreachable states may exist which violate this relation. If these invariants are applied as constraints to the SAT solver in the unrolled timeframes of the circuit, it will not allow the SAT solver to assign the violating unreachable states at the PPIs. Thus, inductive invariants can help to constrain the state space during 
equivalence checking.

\section{Assume-and-verify approach}

The assume-and-verify methodology $[27,64,66]$ uses iterative approach to prove a set of invariants. In this method, we take two timeframes of a circuit (more if cross-timeframe relations are present). For each iteration, in the first timeframe, we assume all the invariants under verification to be true and apply them as constraints. These constraints are very effective to restrict a subset of reachable states. In the second timeframe, we check the validity of the invariants one after another. If an invariant is falsified by the SAT solver, it will be dropped from the assumption in first timeframe. The process is repeated till we reach a fix-point. The remaining invariants at the end will be the list of true invariants.

\subsubsection{Random Simulation and Potential Invariants Generation}

Random simulation allows us to learn a list of potential invariants among the state variables and internal signals in the miter by observing at the relationship between signals on the fly. Both two-node invariants (including cross-timeframe relations) and three-node potential invariants are identified. The relations may be among the variables within the same circuit or between the two circuits under verification.

Let us take three copies of a miter circuit and logic simulate it using $N=8$ random vectors. The assignments for three arbitrary signals $a, b$ and $c$ during 3-bit parallel random simulation 
Table 2.3: Parallel Random Simulation

\begin{tabular}{|c|c|c|c|}
\hline $\mathbf{v}_{i}$ & $\mathbf{a}$ & $\mathbf{b}$ & $\mathbf{c}$ \\
\hline $\mathrm{v}_{1}$ & 111 & 001 & 100 \\
\hline $\mathrm{v}_{2}$ & 001 & 111 & 000 \\
\hline $\mathrm{v}_{3}$ & 101 & 011 & 010 \\
\hline $\mathrm{v}_{4}$ & 110 & 101 & 011 \\
\hline $\mathrm{v}_{5}$ & 111 & 110 & 001 \\
\hline $\mathrm{v}_{6}$ & 010 & 111 & 101 \\
\hline $\mathrm{v}_{7}$ & 100 & 011 & 110 \\
\hline $\mathrm{v}_{8}$ & 000 & 111 & 001 \\
\hline
\end{tabular}

are shown in Table 2.3. These signals may be present in either of the two circuits. We observe that the combination " 00 " between the signals $a$ and $b$ for any vector are missing and we can generate a potential invariant $\left(a_{0} \vee b_{0}\right)$. Therefore, without looking at the database, we can say that the combination " $00 X$ " among the signals a,b and c is also missing where $\mathrm{X}$ is a don't care value. The two newly generated three-node invariants are $\left(a_{0} \vee b_{0} \vee c_{0}\right)$ and $\left(a_{0} \vee b_{0} \vee \neg c_{0}\right)$. Note that these two three-node invariants would be redundant if $\left(a_{0} \vee b_{0}\right)$ is true. However, since all invariants are only potential at this time, we leave all such invariants in. By looking into the table, the three-node assignment "111" for the signals $a, b$ and $c$ is also found to be absent for which the potential invariant generated is $\left(\neg a_{0} \vee \neg b_{0} \vee \neg c_{0}\right)$. Finally, for any value of $i$, the combination " 10 " for the signals $a$ for vector $v_{i}$ and $b$ for vector $v_{i+1}$ are also not found. So, the potential invariant $\left(\neg a_{0} \vee b_{1}\right)$ is generated as a potential cross-timeframe invariant. 


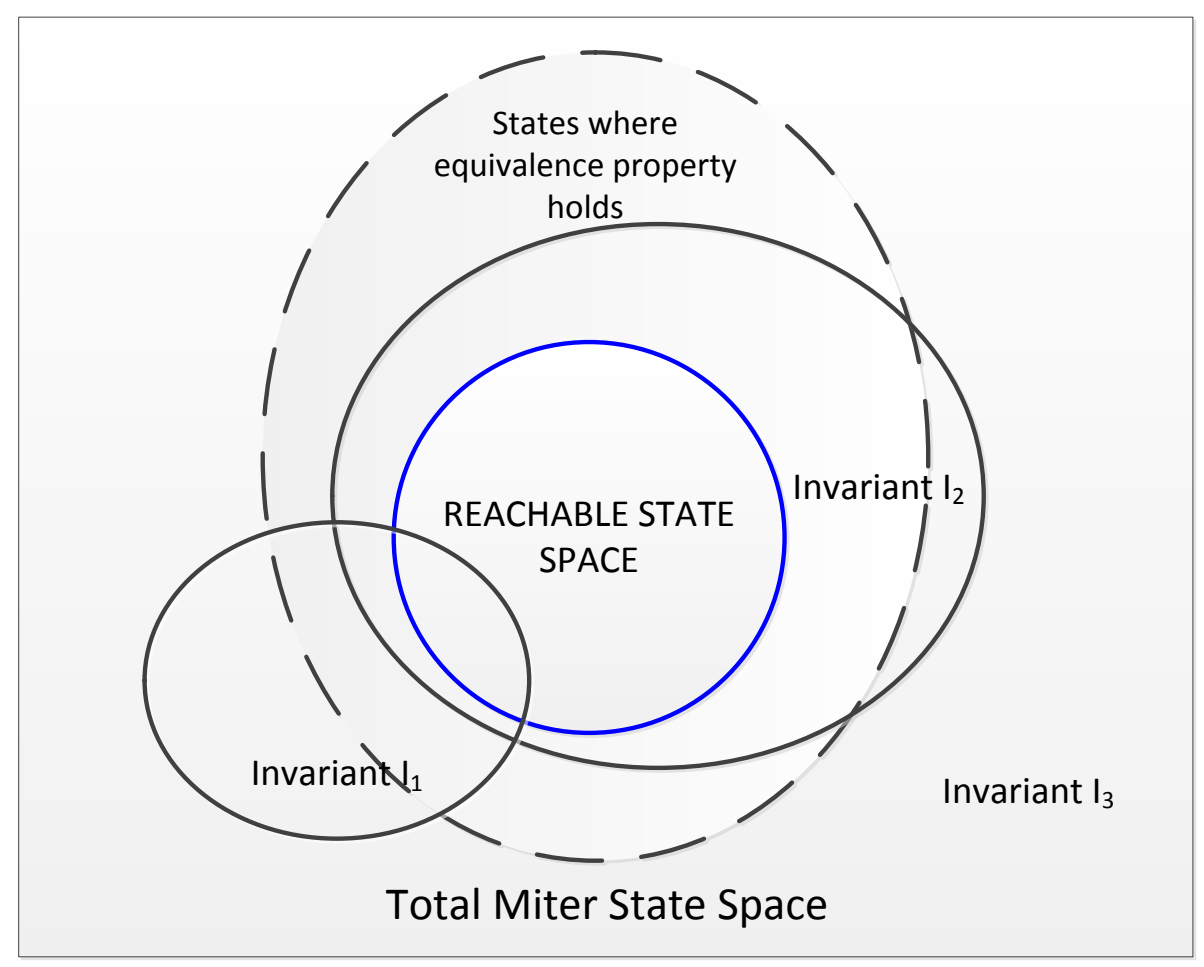

Figure 2.9: Relationship among invariants

Potential invariants involving four or more nodes can result in the exponential rise in the number of invariants, but may be needed eventually to completely constrain unreachable states. Therefore, instead of generating a huge number of potential invariants that may not be able to be handled by SAT solver, two-node cross timeframe relations would be extremely beneficial. Since the random simulation covers a very small reachable state space, only a portion of the potential invariants may really be true. They can be reduced by filtering out those static invariants, which are always true and do not contribute in constraining the search space. 
Consider three potential invariants under verification $I_{1}, I_{2}$ and $I_{3}$ as shown in Fig. 2.9. Invariant $I_{1}$ is true for just a portion of reachable state space, thus it needs to be falsified. Invariants $I_{2}$ and $I_{3}$ are true for the entire reachable state space, but $I_{3}$ is not needed and can be discarded because it is a static invariant and its inclusion does not contribute to constrain any of the illegal states.

\subsubsection{Proving Invariants and Acceleration Techniques}

The set of invariants under verification can be proven using an induction method. In the base case, we take a single timeframe of miter circuit (or two timeframes in case of cross-timeframe invariants of distance ' 1 '), constrain the state variables by a known reachable state and check if the invariant can be violated. For example, if the invariant to be proved is $(a \vee b \vee c)$ which means the signal combination "000" is invalid, we add constraint $(\neg a) \wedge(\neg b) \wedge(\neg c)$. After validating the base case for all the invariants, we proceed with the subsequent induction case. The induction case is based on the assume-and-verify methodology as explained in subsection 2.3.7.

Since the number of invariants under verification may be huge, the SAT solver may take a significant amount of time to prove each invariant for medium to large circuits. There are many static and dynamic acceleration techniques that can be applied to reduce the computation cost significantly. 


\section{Static Invariants Removal}

Before proving the invariants, the static invariants can be identified and dropped since they do not need to be proven and do not help to further constrain the search space. Consider three signals $a, b$ and $c$ in the miter circuit. We first compute direct and indirect twonode implications as explained in Section 2.3.3. To compute three-node implications (same timeframe only), we take a timeframe of miter circuit. If we want to find what $(a, 0)$ and $(b, 1)$ implies, we place the transitive closures of two-node implications of $(a, 0)$ and $(b, 1)$ in the circuit. We make the other gate values unknown and logic simulate the circuit similar to the process of finding indirect two-node invariants. After logic simulation, if we learn that there exists a signal assignment $(c, 1)$, then we can say that $(a, 0)(b, 1) \rightarrow(c, 1,0)$. Conversely, $(a, 0)(c, 0) \rightarrow(b, 0,0)$ and $(b, 1)(c, 0) \rightarrow(a, 1,0)$ are also true.

Dropping static invariants from potential invariants list associated to the learned static invariants is pretty much straight forward. For example, if there exists a static implication $(a, 0) \rightarrow(b, 0, y)$, it means that $(a, 0)$ at timeframe zero and $(b, 1)$ at timeframe $y$ are absent, therefore, its corresponding invariant $\left(a_{0} \vee \neg b_{y}\right)$ can be dropped. If $y=0$, we can also conclude that three-node assignment associated with $(a, 0)(b, 1)$ are automatically absent, e.g. if we consider any variable $q$, the combination " $01 X$ " for signals $a, b$ and $q$ are absent. So, all the three-node invariants associated with $(a, 0)(b, 1)$ can be dropped. If there exists a threenode static implication $(a, 0)(b, 0) \rightarrow(c, 1,0), a b c=000$ are absent, and the corresponding invariant $(a \vee b \vee c)$ can be discarded. 


\section{Dynamic Acceleration Techniques}

In the assume-and-verify method, we assume that all the invariants under verification are true and add them as constraints in first timeframe. Then we check the if each invariant can be falsified in second timeframe. If any invariant is falsified, it should also be dropped from the assumptions in the first timeframe and then only we can proceed to prove the next invariant. But if we remove the falsified invariants from assumptions only at the end of the current iteration, we can save significant amount of time spent while updating the assumptions every time any invariant is falsified.

If an invariant is falsified in an iteration, the SAT solver returns the variable assignment that acts as a counterexample to falsify that invariant. That counterexample may have violated some other invariants as well. By checking the SAT assignments, we can falsify other violated invariants and reduce many SAT calls and iterations as well.

Consider a set of potential invariants as following:

$$
\begin{aligned}
& i_{1}=\left(a_{0} \vee b_{0}\right) \\
& i_{2}=\left(a_{0} \vee b_{0} \vee z_{0}\right) \\
& i_{3}=\left(\neg c_{0} \vee a_{0}\right) \\
& i_{4}=\left(b_{0} \vee d_{0}\right) \\
& i_{5}=\left(\neg c_{0} \vee e_{0}\right) \\
& i_{6}=\left(b_{0} \vee e_{0}\right) \\
& i_{7}=\left(a_{0} \vee f_{0}\right)
\end{aligned}
$$


Let us say the invariant $i_{1}$ be proven true in the current iteration and $i_{2}$ has not been not evaluated yet. Since the space constrained by $i_{1}$ is superset of the space constrained by $i_{2}, i_{2}$ is definitely going to be true when $i_{1}$ is true and thus we do not need to evaluate $i_{2}$ in the current iteration and can be skipped. Suppose there exists a static logic implication $(c, 1) \rightarrow(b, 0,0)$. Since $i_{1}$ can be represented as $(b, 0) \rightarrow(a, 1,0)$, we can derive $(c, 1) \rightarrow(a, 1,0)$ by law of transitivity, and thus the related invariant $i_{3}$ can be declared to be true automatically for this iteration and skipped. Similarly, if there exists another static implication $(e, 0) \rightarrow$ $(a, 0,0),(e, 0) \rightarrow(b, 1,0)$ and thus $i_{6}$ can be skipped. Moreover, since $(c, 1) \rightarrow(b, 0,0)$ from assumption and $(b, 0) \rightarrow(e, 1,0)$ from $i_{6},(c, 1) \rightarrow(e, 1,0)$ by law of transitivity and thus $i_{5}$ can be skipped in this iteration.

Let us say $i_{1}$ and $i_{4}$ are proved to be true in current iteration. After placing all the static implications in transitive closure of $(a, 1)$ plus newly learned implications representing already evaluated invariants related to $(a, 1)$ and logic simulating, consider a new node $f$ be evaluated to be logic ' 0 '. $(a, 1) \rightarrow(f, 0,0)$ is newly formed implication and therefore, $i_{7}$ can be skipped in this iteration.

\subsubsection{Sequential Equivalence Checking Framework}

The flowchart in Fig. 2.10 illustrates the sequential equivalence checking method [27]. For a timeframe $k, I_{k}$ represents the primary input, $O_{k}$ represents the final output and Inv represents the list of true invariants at timeframe $k$. 


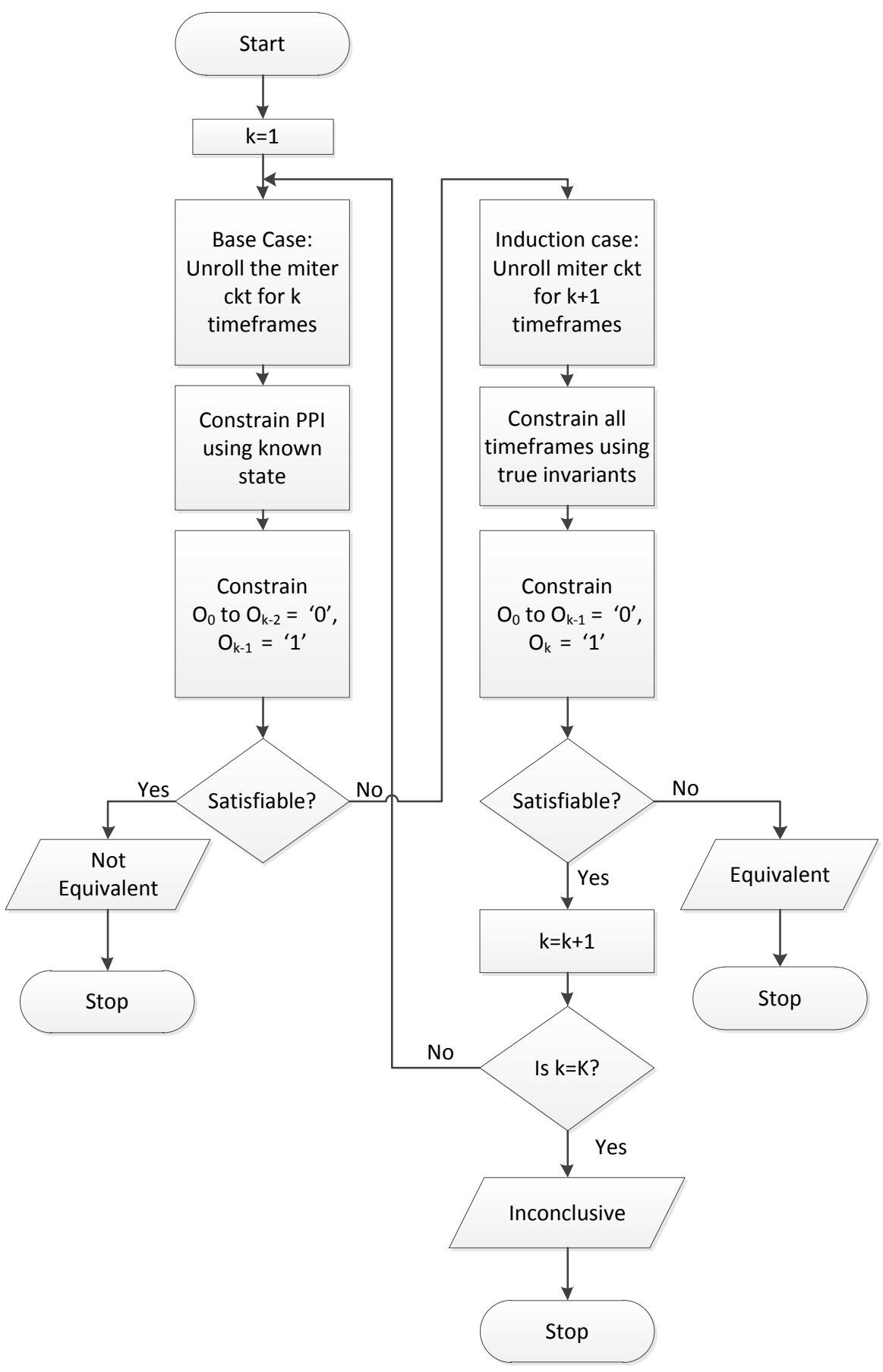

Figure 2.10: Flowchart for Sequential Equivalence Checking 


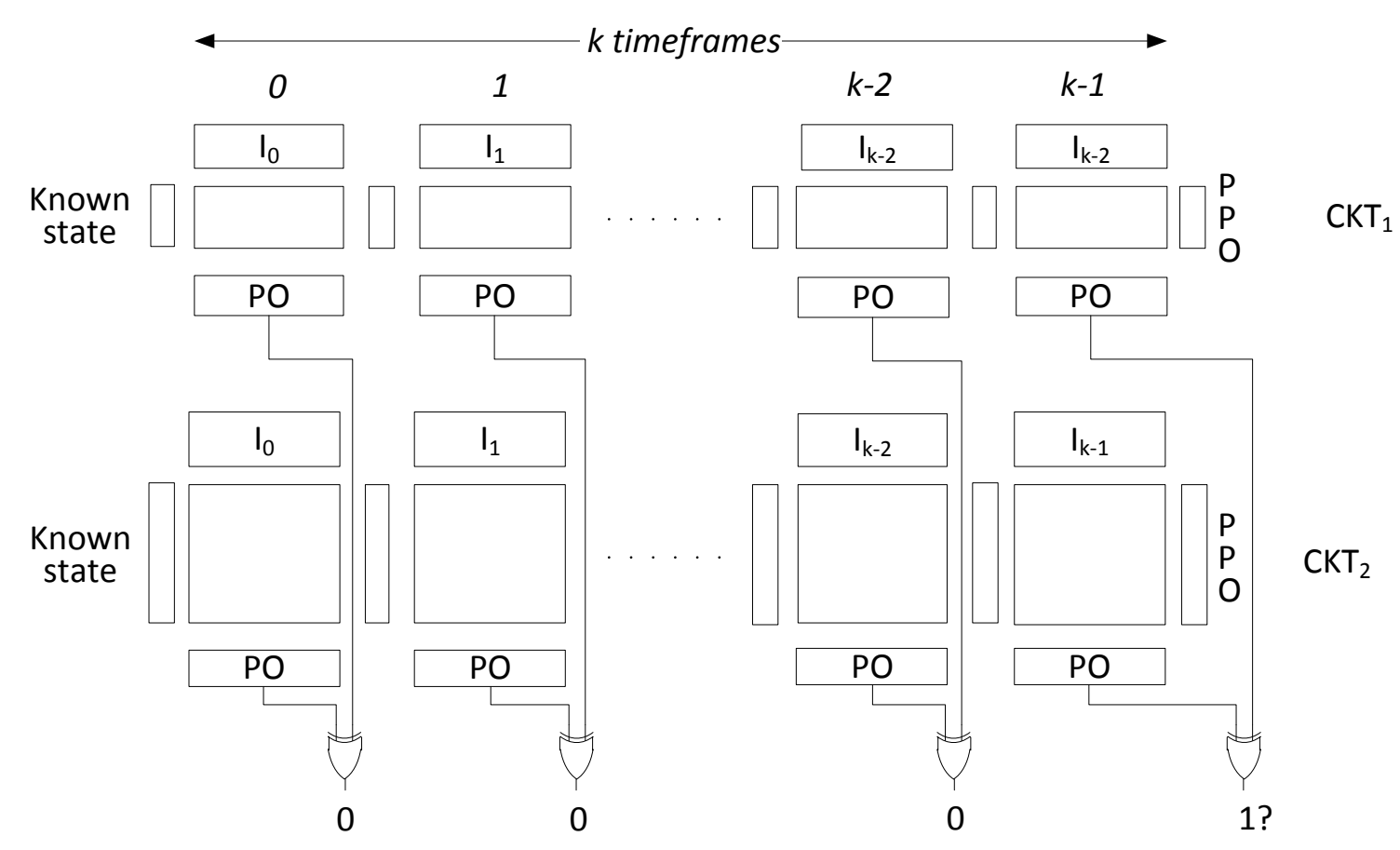

Figure 2.11: Base Case for Sequential Equivalence Checking

In the base case, we unroll the miter circuit for $k$ timeframes within the unroll threshold $K$ where $k$ varies from 1 to $K$, and constrain the pseudo-primary input (PPI) by the known reachable state as shown in Fig. 2.11. We apply the true invariants in all timeframes and force the final outputs $O_{0}$ to $O_{k-2}$ to logic ' 0 '. We check if the equivalence property is violated at timeframe $k-1$ by constraining the final output $O_{k-1}$ to be logic ' 1 '. If the SAT solver returns a satisfiable assignment, it acts as the counterexample to distinguish the two circuits and thus two circuits can be concluded as inequivalent. If the SAT solver returns unsatisfiable, we proceed to the induction step.

In the induction case, we take $k+1$ timeframes of the miter circuit as shown in Fig. 2.12. 


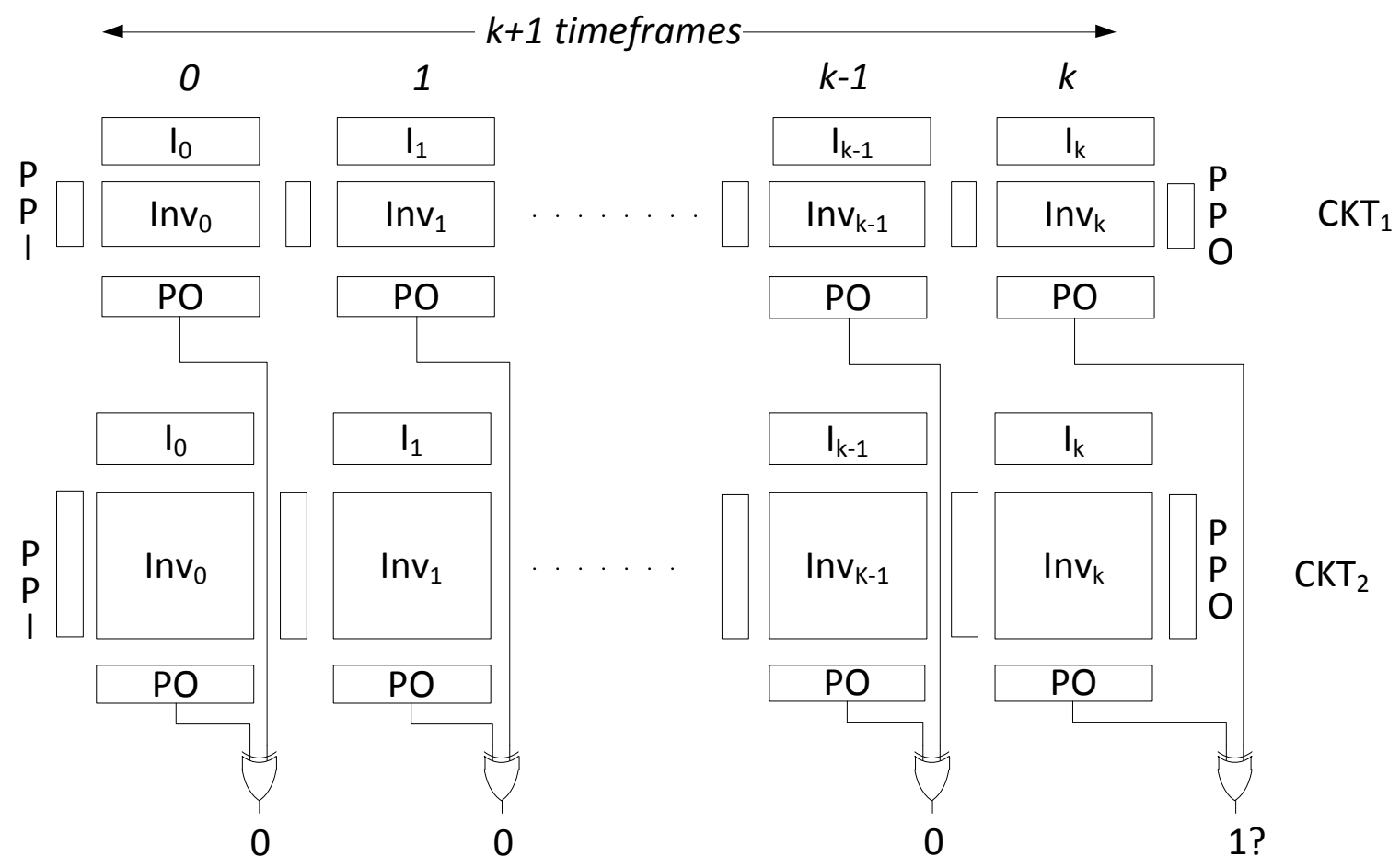

Figure 2.12: Induction Case for Sequential Equivalence Checking

We do not apply reachable state at PPI but instead apply true invariants at all timeframes. We assume that the equivalent property holds for timeframes 0 to $k$ - 1 by forcing the final outputs $O_{0}$ to $O_{k-1}$ to be logic ' 0 '. The final output $O_{k}$ is forced to logic ' 1 ' to check if the equivalence property is violated at timeframe $k$. If the SAT solver returns unsatisfiable, it means there is no reachable state that can violate the equivalence property and thus can be concluded as equivalent. If the $S A T$ solver returns satisfiable, we cannot conclude about equivalence because the satisfiability may be due to unreachable state at the timeframe $k$. In this case, we increment $k$ by 1 , repeat the iteration process from base case again till $k$ reaches unroll threshold $K$. If unroll threshold is reached, no conclusion is drawn about the equivalence. 


\section{Chapter 3}

\section{Test Generation and Equivalence}

\section{Checking}

This chapter presents our main contribution where we attempt to ensure the trust of thirdparty IPs. We use two-step methodology to declare about the behavior of suspicious signals in the suspect circuit. In the first step, we activate and propagate the suspicious signal to the primary output by using the miter circuit constructed from two copies of the suspect circuit and injecting a fault side in one timeframe of one copy of the circuit. If we get a counterexample that activates the suspicious signal and propagates its effect to the primary output, we proceed to the second step. In the second step, we use the counterexample to check if the propagated activation in the suspect circuit is observed in the spec circuit as well, via a constrained sequential equivalence checking setup. 


\subsection{Motivation}

A few pre-silicon Trojan detection methods are based on test generation and formal equivalence checking. But those techniques may not be complete or optimal. For instance, in [51], the authors make the primary outputs of the designs as functions of only primary inputs during equivalence checking. However, in most of the designs, it is impossible to make the outputs independent of the state variables. For such cases, the reachability of the state variables should be addressed to ensure the efficient detection of the malicious inclusions. In [52], the authors construct the triple miter for equivalence checking using the two copies of suspect circuit and a copy of spec circuit as mentioned in the previous chapter. But it may increase the problem size unnecessarily as the number of variables are huge for big designs, resulting in tremendous increase in the search space. Hence, the use of a traditional miter circuit (containing only two circuits instead of three) would be a better solution. Nevertheless, one should note that SEC is a difficult problem, especially when the spec and suspect circuits differ drastically, in terms of the number of gates and state variables. They do not constrain initial state of the miter circuit during equivalence checking which makes the method less efficient. But there are methods to generate invariants that can constrain the state spaces efficiently. Therefore, methods to reduce this complexity and the search space during the search are necessary to make SEC-based approaches feasible for Trojan detection.

We assume that the Trojan has been implanted in the RTL or gate level by an unknown adversary and the design is available to us in the netlist form. We also expect the Trojan 
to be minute (one or few gates), trigger on rare internal signal conditions and change the functionality of circuit. The objective is to guarantee that either the design is trusted or identify the Trojan signals in the design.

\subsection{Random Simulation and Equivalence Checking}

As discussed earlier, formal and semi-formal (combination of both simulation-based and formal) equivalence checking methods need a reference circuit called golden model to verify the functionality of the Circuit Under Verification (CUV). Golden model is available easily in the trusted design environment. However, for an untrusted IP, the availability of an ideal golden model is a big question. One way to obtain the golden model is to prepare an unoptimized and quickly synthesized circuit from the specifications [52]. This can be done by a fully trusted third party or by the buyer oneself. The other way is to use a second untrusted third-party IP assuming that even if both circuits have malicious inclusions, the chances of both Trojans being at the same place, and having same trigger conditions and same functional effect is very unlikely [51]. Our technique assumes that the reference circuit is an unoptimized (potentially much larger) circuit free from malicious insertions prepared in a trusted environment.

We construct a miter circuit using the untrusted IP core as a suspect circuit and a reference spec circuit as shown in Fig. 3.1. One should note that the two circuits may be drastically different in terms of gates and flip-flops count without any or with little structural similari- 


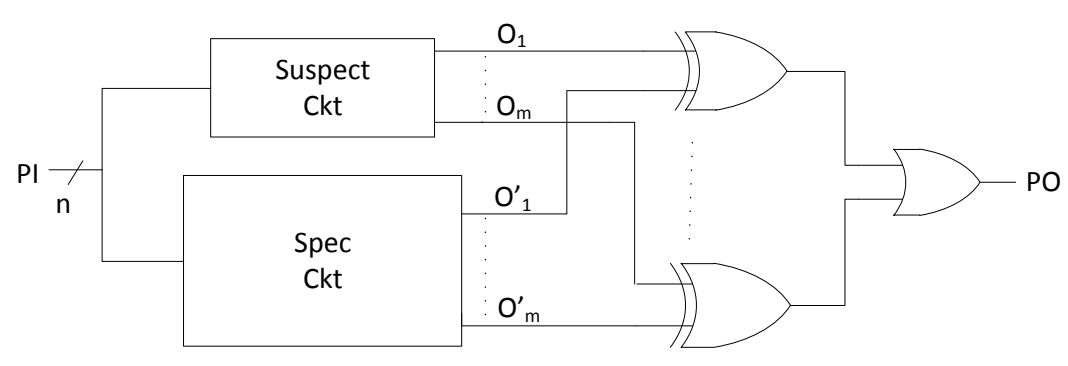

Figure 3.1: Miter circuit with suspect and spec circuits

ties. Traditional equivalence checking that relies on structural similarities $[64,65]$ would be ineffective in such cases. The size of the suspect circuit is smaller than the size of the spec circuit because suspect circuit is very optimized compared to spec circuit. Next, we logic simulate the miter circuit using $N$ random input test vectors. There are two objectives of the random simulation: (1) Suspect Signals Generation, and (2) Invariants Generation.

\subsubsection{Suspect Signals and Inductive Invariants Generation}

During simulation, we keep track of switching activities (toggles) of the internal signals in the suspect circuit for the instances in which the state variables are fully specified. Then we divide all the internal signals of the suspect circuit into suspicious and non-suspicious signals. Suspicious signals are identified as those signals which do not flip much during logic simulation. Non-suspicious signals, on the other hand, do not mean that they are not Trojan signals rather they are less likely compared to suspicious signals because of the stealthy nature of the Trojan. Therefore, we can discard non-suspicious signals to narrow 
down the search for the malicious signals.

The signals under consideration are the gate outputs in the circuit. The output of singleinput gates (NOT, BUFFER, D flip-flop) do not need to be considered because their toggling frequency is same as the toggling frequency of their driving signal. The set of $p$ suspicious signals, which have less toggle frequencies during logic simulation, in the suspect circuit is given by, $S=\left\{S_{1}, S_{2}, S_{3} \ldots S_{p}\right\}$. Random Logic simulation of the miter circuit can also be used to generate potential invariants as discussed in Section 2.3.8. Section 2.3.9 describes the method to prove invariants under verification. The true invariants can be extremely useful to prove equivalence of two circuits having completely different internal structures as shown in Section 2.3.10.

\subsubsection{Application of SEC to the Miter}

Over-approximation based sequential equivalence checking using SAT solver (as discussed in Section 2.3.10) is used to verify if suspect and spec circuits are equivalent. The SEC will declare the two circuits as (a)equivalent (b) inequivalent or (c) inconclusive.

\section{Equivalent}

If the SEC declares that the two circuits are equivalent, we can conclude that the suspect circuit shows exactly the same output behavior as it should at any reachable state. Therefore,

even though a Trojan circuit(s) is present in the suspect circuit, it won't be able to propagate 
its effect to any of the primary outputs. Thus, we can ensure the trust of the suspect IP. One should note that even though the two circuits are functionally equivalent, we cannot avoid the possibility of any other non-functional physical behavior such as leaking key information of a cryptographic hardware via side channel power during the circuit operation.

\section{Inequivalent}

If the suspect and spec circuits are declared inequivalent, we obtain a counterexample that differentiates the two circuits. We save the reachable state in the particular timeframe where the outputs of two circuits are different. This helps in bringing the effect of suspicious signals to this state in further analysis.

\section{Inconclusive}

If the true invariants are stated inconclusive, the invariants under consideration are not enough to prove equivalence (if the two circuits are indeed equivalent). On the other hand, we also cannot avoid the possibility of Trojan insertions in the suspect circuit since the circuits may not be equivalent. Therefore, we cannot ensure the trust of the suspect circuit.

For the latter two declarations (Inequivalent and Inconclusive), we need to check the effect of activation of suspicious signals in the output behavior of suspect circuit. So, we activate a suspicious signal, propagate it to the primary output and formally prove that the output behavior is malicious. 
Gyanendra Shrestha

\subsection{Detection of Malicious Signals}

Considering the case in which the two circuits have not been proven equivalent, they can be either Inconclusive or Inequivalent as explained in the above discussion. From the set of $p$ suspicious signals, we need to identify the signals which are most likely to show the malicious behavior when they are excited. For any value of $i$, where $1 \leq i \leq p$, we propose two-step methodology to declare about the behavior of the suspicious signal $S_{i}$ :

(a) Invariant Based Test Generation

(b) Counterexample Guided Equivalence Checking

\subsubsection{Invariant-Based Test Generation}

The idea behind invariant-based test generation is the concept of test generation for fault detection. To detect a fault, a fault should be excited and propagated to the primary output. Similarly, to say something about the effect of a suspicious signal, we first need to activate the suspicious signal and find a path to propagate its effect to at least one of the primary outputs. The signal $S_{i}$ is said to be activated when we get the rare logic value at that signal in the circuit. We can propagate the effect of the signal activation to the primary output by finding a sensitization path starting from the point of activation to the primary output which may even take several timeframes. The fundamentals of path sensitization is explained in Section 2.3.2. 
We first create a miter circuit using two identical copies of the suspect circuit unrolled for $k$ timeframes where $1 \leq k \leq K$. Note that no spec circuit is used here. We can simply tie the initial pseudo-primary input pairs of the two circuits since both circuits are identical copies. We inject a stuck-at fault on the suspicious signal in only one timeframe of one copy of the circuit. We assume that there is no fault in the rest of the timeframes in both the faulty copy (circuit where fault is injected) and the clean copy of the suspect circuit. This makes our method different from the traditional ATPG methods, where the fault is present at all unrolled timeframes.

The target suspect signal $S_{i}$ needs to be activated in a timeframe and its effect is propagated to the primary output within the given unroll threshold $K$. We make sure that the activation effect gets propagated to the output, not to PPO. This is because, in later stages, when we check its equivalence with spec circuit using the counterexample obtained in this step, we cannot compare the PPO logic values of the suspect circuit with that of the spec circuit since they may have different numbers of flip-flops with no resemblance at all. Since we know that a suspect signal may be activated at any reachable state, we cannot constrain the left-most timeframe by a known reachable state. One way to restrict a portion of unreachable state space is to use the true invariants related to the suspect circuit only in its clean copy. We cannot apply these invariants in the faulty copy of the suspect circuit because the signal relations might have changed because of the fault injection and may violate some of the invariants. 


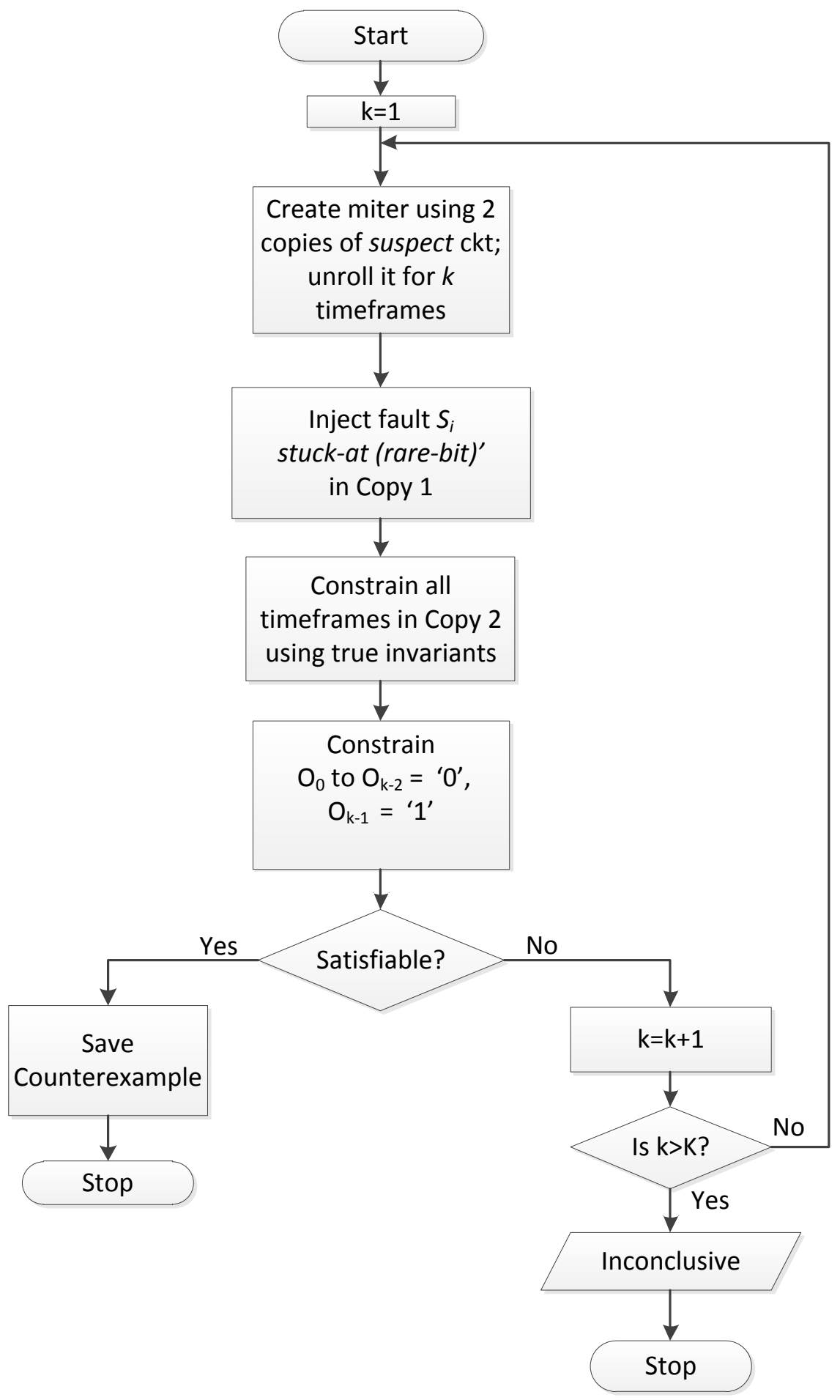

Figure 3.2: Flowchart for Invariant based Test Generation 
Fig. 3.2 shows the flowchart of our invariant-based test generation approach. Let us assume that $I_{k}$ is the miter input, $O_{k}$ is the final miter output and $I n v_{-} S u s_{k}$ is the list of true invariants.

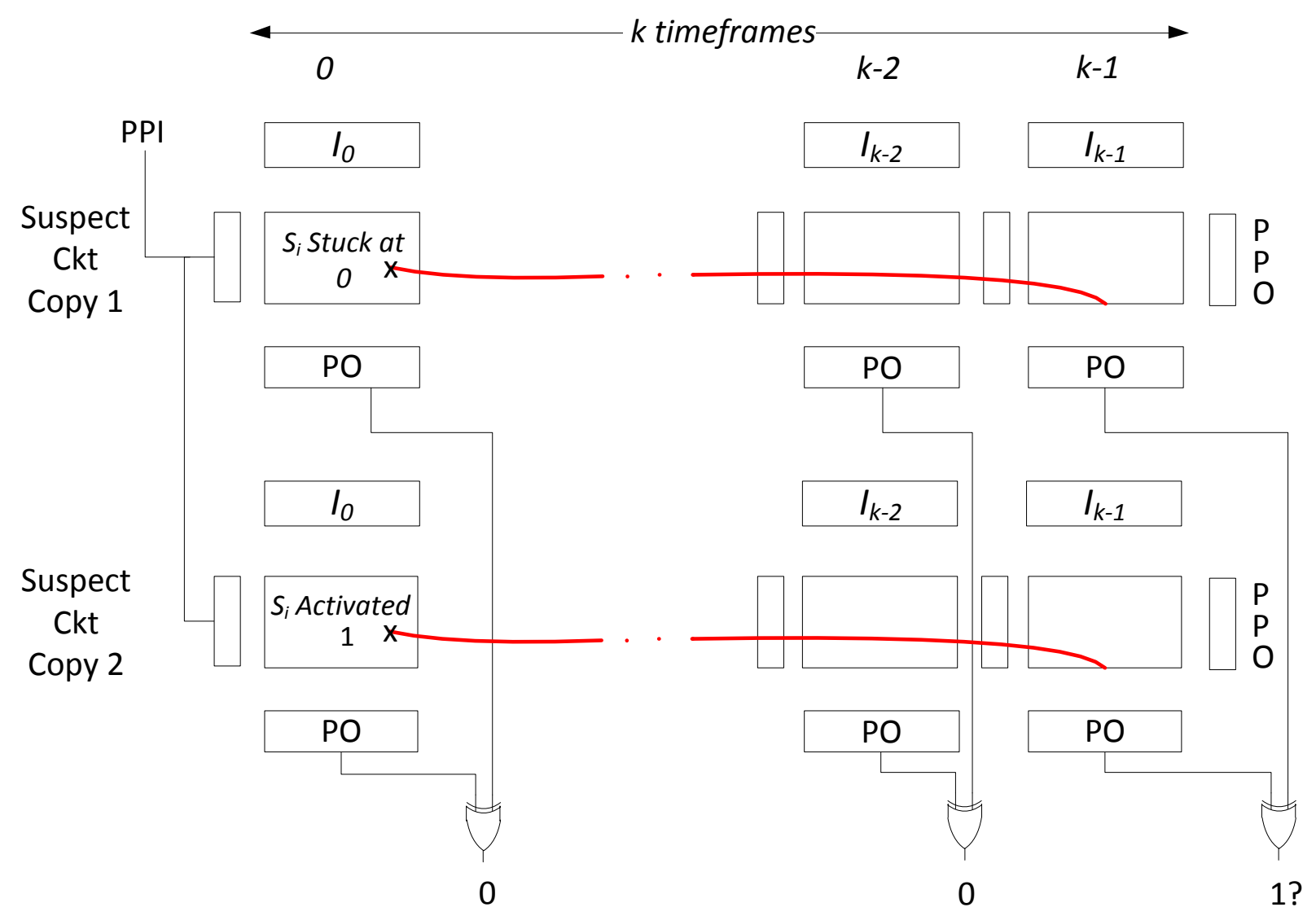

Figure 3.3: Invariant based Test Generation

The setup for invariant based test generation is illustrated in Fig. 3.3. We start with an initial unroll depth $k=1$. We want to check if the non-equivalence is due to the suspect signal. To do so, we need to set a logic value rare-bit at the fault site $S_{i}$, and the stuck-at fault is $S_{i}$ stuck-at $(\text { rare-bit })^{\prime}$. We inject the stuck-at fault at timeframe 0 . Each timeframe 


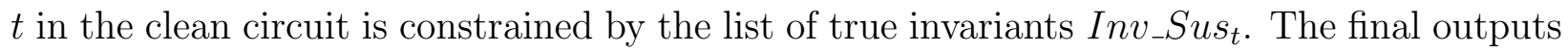
$O_{0}$ to $O_{k-2}$ are forced to be logic ' $O$ '. We check if the two circuits under verification can be distinguished at timeframe $k-1$ by constraining $O_{k-1}$ to logic ' 1 '. If the suspect and spec circuits were declared inequivalent in an earlier step, we apply the known reachable state in which the two circuits were distinguished as a constraint in the clean copy at timeframe $k-1$. We give the propositional formula to the SAT solver to check if there exists any state and the input vector in the suspect circuit that can activate the suspicious signal and propagate its effect to the primary output. If the SAT solver returns a satisfying assignment, it means that the fault is excited at timeframe 0 and its effect is propagated through the sensitized path to the $\mathrm{PO}$ at $k-1$ in the faulty copy. Meanwhile, in the clean copy of the suspect circuit, due to the assignment rare-bit at timeframe 0 , the suspicious signal is activated and its effect is propagated to the PO at timeframe $k-1$ through the identical sensitized path. The starting state, input vector and the node assignments along sensitized path serve as a witness to the activation and the propagation of the suspicious signal and thus are extremely useful. These values in the clean copy are saved as a counterexample to narrow down the search space for SEC in the next step. On the other hand, if the SAT solver cannot give any satisfying solution, $k$ is incremented by 1 and the process is repeated till $k=K$. If there is no satisfying solution at all, we conclude that either the signal cannot be activated and propagated to the primary output in any reachable state (benign signal), or the given unroll threshold is not enough to propagate the activated signal to the output. In such case, we cannot declare about this suspicious signal. 


\section{Strengthening the method}

If we get an counterexample from the step above, we cannot discard the possibility of the signal activation state in the counterexample to be unreachable because the invariants may not have restricted entire unreachable states. But we can increase the probability of the signal activation state to be reachable by adding a number of timeframes $K^{\prime}$ before activating the suspicious signal and propagating its effect to the output and applying the related invariants in the added timeframes. Because of addition of the timeframes with invariants, even if the left-most timeframe is assigned unreachable state, because of constraints, it may transit into reachable state till it reaches the activation timeframe $K^{\prime}$. Once it enters into reachable state space, the next timeframe is always reachable [27]. Fig. 3.4 shows the reachable state space of miter circuit with and without the addition of $K^{\prime}$ timeframes.

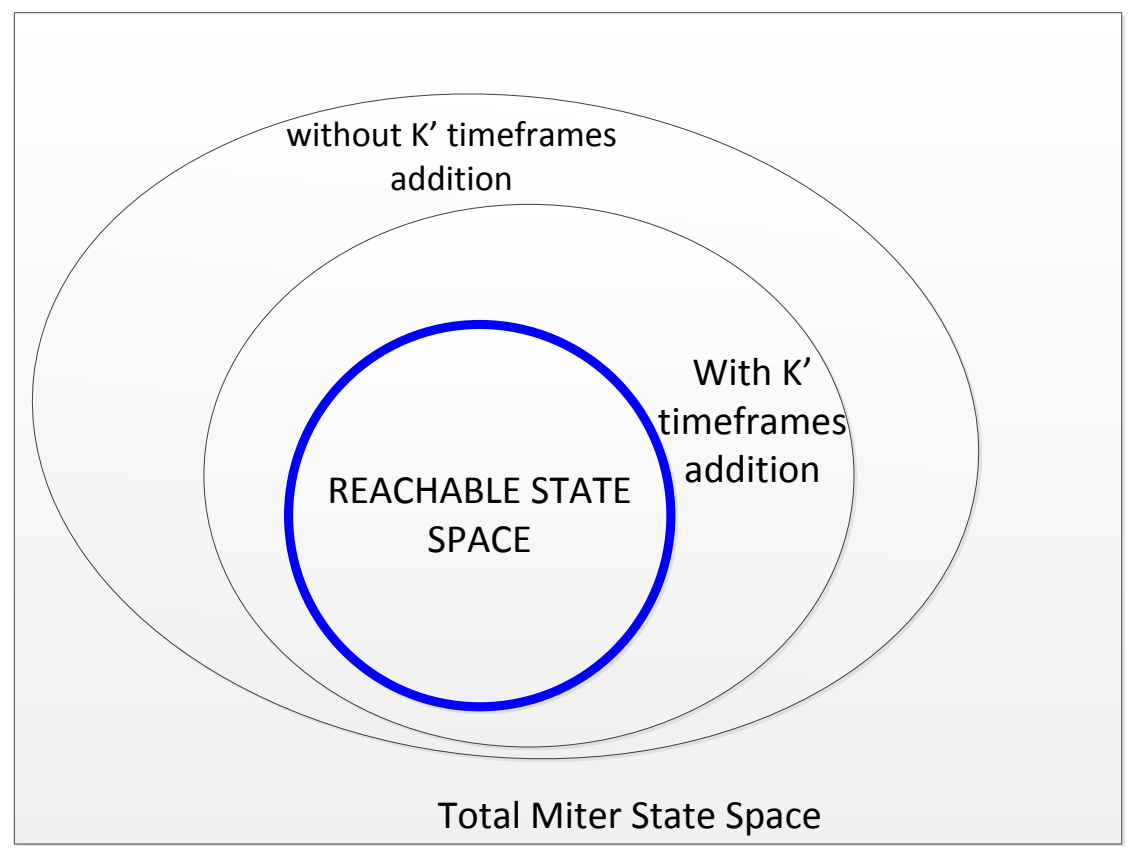

Figure 3.4: State space constraining with the addition of $K^{\prime}$ timeframes 


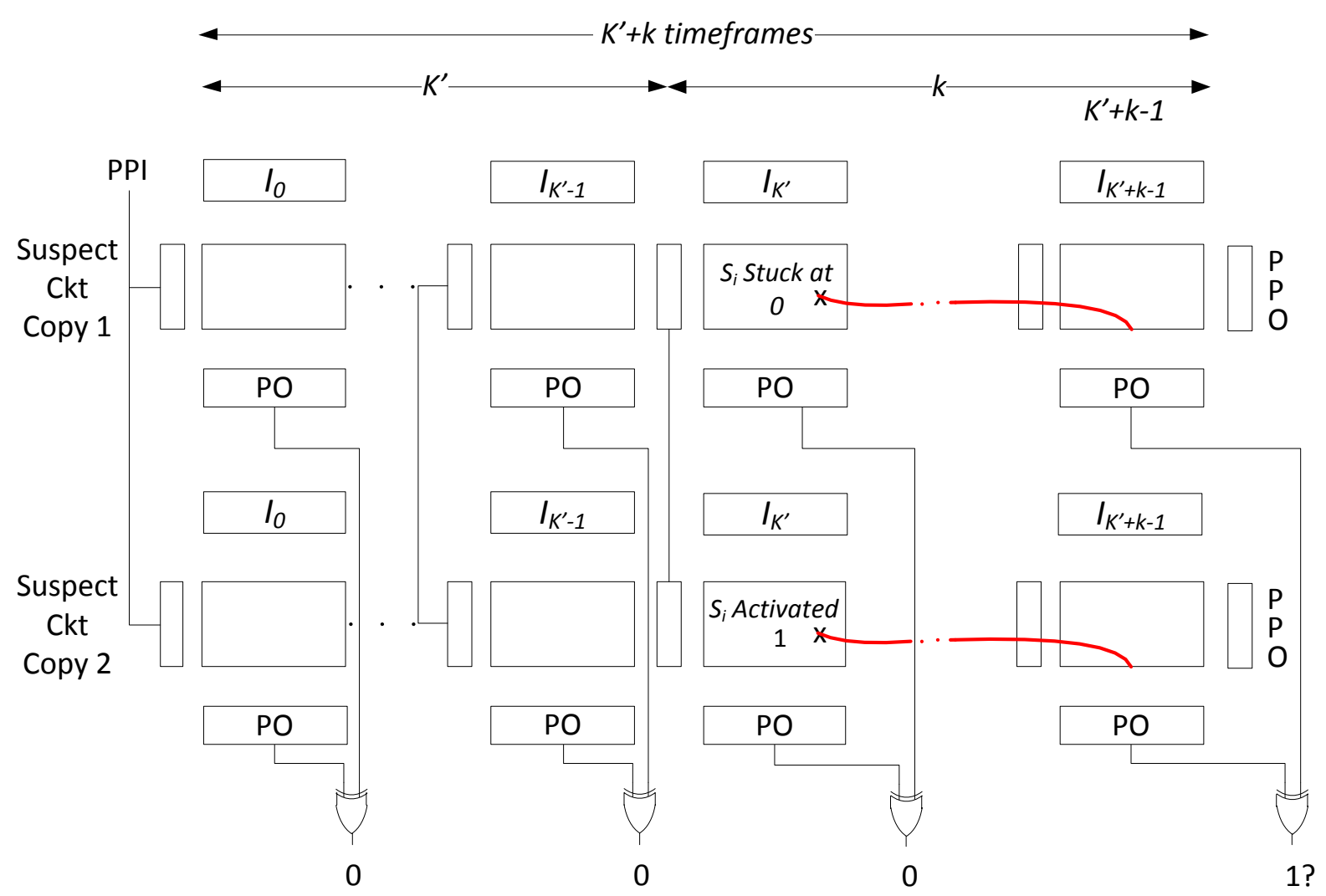

Figure 3.5: Strengthening the Test Generation Method

The setup for strengthening the test generation method is illustrated in Fig. 3.5. The converted flip-flop (either PPI or inter-timeframe buffer) pairs in the miter can be tied till the activation timeframe $K^{\prime}$ because the two circuits are identical till that point. For each timeframe $t$ where $0 \leq t \leq\left(K^{\prime}+k-1\right)$, we add invariant constraint $I n v_{-} S u s_{t}$ to constrain the search space. The final outputs $O_{0}$ to $O_{K^{\prime}+k-2}$ are forced to be logic ' $O$ ' and we check if the outputs can be distinguished at timeframe $K^{\prime}+k-1$ by forcing $O_{K^{\prime}+k-1}$ to be logic ' 1 '. 


\subsubsection{Counterexample Guided Equivalence Checking}

The counterexample obtained in subsection 3.3.1 provides a propagation sequence for the activated target suspect signal. However, it does not guarantee that the activation effect of suspicious signal under consideration is malicious. In this step, we check if that activation effect is really malicious. Fig. 3.6 shows the flowchart of our counterexample guided sequential equivalence checking approach.

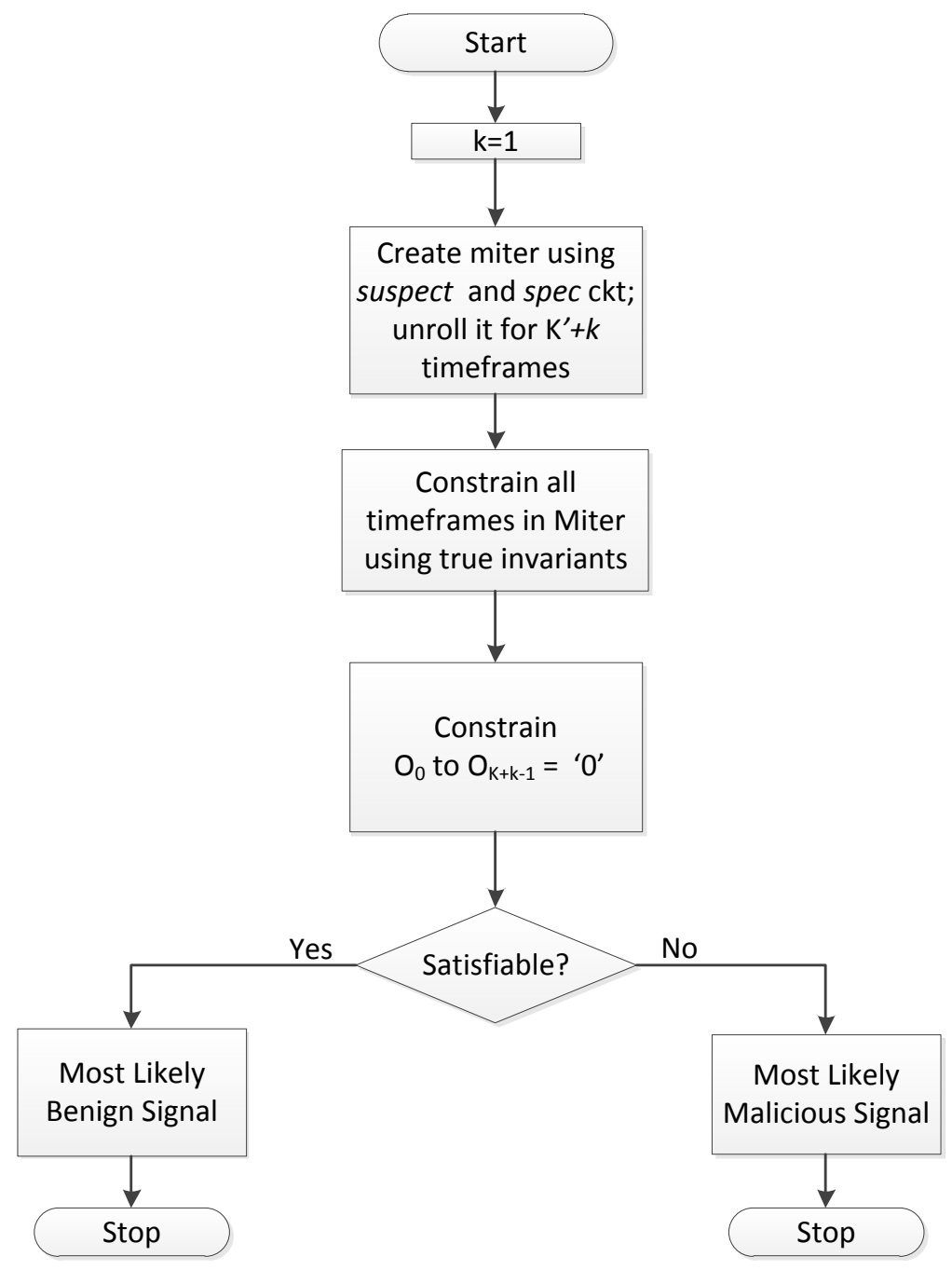

Figure 3.6: Flowchart for Counterexample Guided Equivalence Checking 

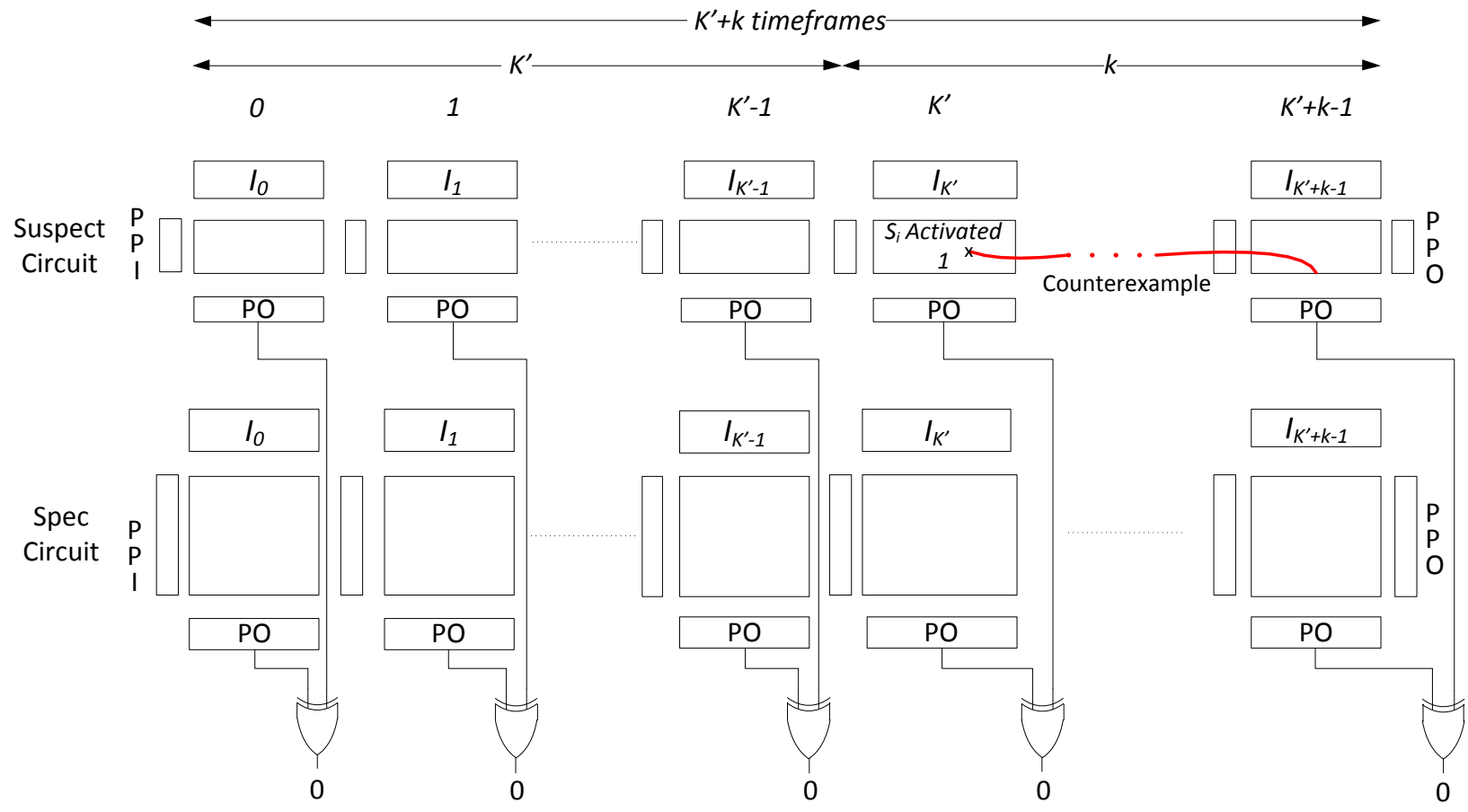

Figure 3.7: Counterexample guided Equivalence Checking

Fig. 3.7 illustrates the setup for this counterexample guided equivalence checking method. For any timeframe $t, I_{t}$ is the miter input, $O_{t}$ is the miter final output and $I n v_{t}$ is the list of all true invariants. We create a miter circuit using the clean copy of the suspect circuit and the spec circuit, and unroll it for $K^{\prime}+k$ timeframes. The true invariants are applied to this miter circuit (consisting of suspect and spec circuits) at all timeframes to constrain the large subset of unreachable state space. The final primary outputs at all timeframes, $O_{0}$ to $O_{K^{\prime}+k-1}$ are constrained to logic ' 0 '. As mentioned in subsection 3.3.1, if we add true invariants at all these additional $K^{\prime}$ timeframes and force the final outputs $O_{0}$ to $O_{K^{\prime}-1}$ to logic ' $O$ ', it increases the probability of the activation timeframe to be reachable. 
Let us say the suspicious signal $S_{i}$ in the suspect circuit is activated in $\left(K^{\prime}\right)^{\text {th }}$ timeframe and is detected in $\left(K^{\prime}+k-1\right)^{t h}$ timeframe as in subsection 3.3.1. Now we add the node assignments along the sensitized path starting from activation point of suspicious signal $S_{i}$ at timeframe $K^{\prime}$ to the primary output at timeframe $K^{\prime}+k-1$ obtained from subsection 3.3.1 in the suspect circuit. This setup makes sure that the suspect signal is activated and the effect of the activation is propagated through sensitized path to the primary output. If the suspicious signal is malicious and the sensitized path propagates the malicious effect to the output of the suspect circuit, there exists no state in the spec circuit that can show same behavior as the malicious output in the suspect circuit. The propositional formula is given to the SAT solver to check if there exists any state in the spec circuit that can show the same output behavior as the suspect circuit in which the suspicious signal has been activated and its effect has been propagated to the primary output.

Since the miter output is constrained to ' $O$ ' at all timeframes, the spec circuit must give identical output behavior as the suspect circuit for all the reachable state pairs possible. Hence, if the instance is satisfiable, the suspicious signal $S_{i}$ would most likely be benign since the malicious nature is not present in the spec circuit for all reachable states. On the other hand, if the instance is unsatisfiable, it means that the spec circuit cannot produce the same outputs as the suspect circuit with the path confined by the counterexample, indicating that the suspect signal is likely to be malicious. 
Gyanendra Shrestha

\subsection{Novelty of the Approach}

Conventional test generation methods are used to generate test vectors for detection of manufacturing defects in the ICs. In manufacturing test, a target fault would be a defect on an existing signal, while a malicious Trojan may be an addition of logic. Thus, we cannot apply such methods directly for detection of malicious Trojans. Even if we guarantee the $100 \%$ fault coverage by existing ATPG tools, the test vectors may not be enough to identify hard Trojans inserted in the design. However, test generation does have its place. We use a test generation method for the concept of fault excitation and propagation to activate and propagate the suspicious signal; but in our case, the fault is present only in the suspect signal activation timeframe. Moreover, the use of inductive invariants during sequential equivalence checking has been proven to be very effective in constraining the search space during SAT solving [27]. The search space of the proposed test generation technique using SAT can also be reduced by restricting the subset of unreachable states and allowing most likely reachable state for activation of suspicious signal.

During counterexample guided equivalence checking, the addition of the counterexample obtained in the test generation stage guarantees the activation and propagation of the suspect signal. Thus, we are asking if the spec circuit can produce the same behavior under the same counterexample that had activated and propagated the suspect signal in the sus circuit. In addition, as the two circuits under verification (suspect and spec circuits) may differ structurally, the use of inductive invariants in both of the circuits to constrain the search 
Gyanendra Shrestha

space makes our approach unique from the rest of the Trojan detection techniques.

\subsection{Experimental Results}

Our proposed detection methodology was implemented in $\mathrm{C}++$ with an Intel Core-i5, 2.5 GHz PC, 4 GB RAM running 32-bit Ubuntu 12.04. A suite of hard-to-verify SEC benchmarks [27] was constructed from gray and one hot encodings of ITC99 benchmark circuits. The two circuits in SEC benchmarks had different number of flip-flops and gate counts with very few or no internal equivalent nodes.

Very minute and hard Trojans were created by selecting the points having minimum switching activities during logic simulation of original design by adding inverter(s), deleting inverter(s) or changing the gate type. After Trojan insertion, we performed parallel logic simulation in suspect benchmark miters using 100,000 random vectors altogether. We dropped those Trojans that logic simulation was enough to distinguish the two circuits to make sure it was not easily detectable (stealthy nature). During the simulation process, we also identified potential invariants and separated signals into suspicious and non-suspicious groups. The maximum number of suspicious signals was limited to 500. The unroll depths $K$ and $K^{\prime}$ of miter circuit were both limited to 10 . However, these could be easily adjusted. We used Zchaff [58] as the SAT Solver which has incremental SAT solving feature; however, we note that other solvers may be used as well.

Table 3.1 presents results for different benchmark miter circuits with Trojan inserted in 
Table 3.1: Results for Equivalence Checking of Hard SEC Benchmark Circuits

\begin{tabular}{|c|c|c|c|c|c|c|c|c|}
\hline \multirow{2}{*}{ Name of Ckt } & \multirow[t]{2}{*}{$\mathrm{I} / \mathrm{O}$} & \multirow[t]{2}{*}{$\mathrm{FF}$} & \multirow{2}{*}{$\begin{array}{c}\text { Trojan } \\
\# \\
\end{array}$} & \multirow{2}{*}{ Inductive Inv. } & \multirow{2}{*}{$\begin{array}{c}\text { \# Sus } \\
\text { Sig. }\end{array}$} & \multicolumn{3}{|c|}{ Equiv. Checking } \\
\hline & & & & & & Eqv & N.E. & I.C. \\
\hline \multirow{5}{*}{ b01_gray_onehot } & \multirow{5}{*}{$4 / 2$} & \multirow{5}{*}{$5 / 10$} & 1 & $1594 / 87 / 1089$ & 40 & - & - & $\checkmark$ \\
\hline & & & 2 & $1594 / 87 / 1089$ & 40 & - & - & $\checkmark$ \\
\hline & & & 3 & $1564 / 87 / 1089$ & 40 & - & - & $\checkmark$ \\
\hline & & & 4 & $1594 / 87 / 1089$ & 40 & - & - & $\checkmark$ \\
\hline & & & 5 & $1589 / 87 / 1089$ & 42 & - & - & $\checkmark$ \\
\hline \multirow{3}{*}{ b02_gray_onehot } & \multirow{3}{*}{$2 / 1$} & \multirow{3}{*}{$4 / 8$} & 1 & $570 / 74 / 652$ & 16 & - & - & $\checkmark$ \\
\hline & & & 2 & $570 / 74 / 652$ & 17 & - & - & $\checkmark$ \\
\hline & & & 3 & $541 / 73 / 641$ & 17 & - & - & $\checkmark$ \\
\hline \multirow[b]{2}{*}{ b03_gray_onehot } & \multirow[b]{2}{*}{$6 / 4$} & \multirow[b]{2}{*}{$30 / 31$} & 1 & $34424 /-/-$ & - & $\checkmark$ & - & - \\
\hline & & & 2 & $34436 /-/-$ & - & $\checkmark$ & - & - \\
\hline \multirow[b]{2}{*}{ b06_gray_onehot } & \multirow[b]{2}{*}{$4 / 6$} & \multirow[b]{2}{*}{$9 / 13$} & 1 & $148 /-/-$ & - & $\checkmark$ & - & - \\
\hline & & & 2 & $148 /-/-$ & - & $\checkmark$ & - & - \\
\hline \multirow{7}{*}{ b08_gray_onehot } & \multirow{7}{*}{$11 / 4$} & \multirow{7}{*}{$21 / 23$} & 1 & $18341 / 0 / 0$ & 132 & - & $\checkmark$ & - \\
\hline & & & 2 & $2704 / 0 / 0$ & 132 & - & $\checkmark$ & - \\
\hline & & & 3 & $18303 / 0 / 0$ & 132 & - & $\checkmark$ & - \\
\hline & & & 4 & $1973 / 0 / 0$ & 135 & - & $\checkmark$ & - \\
\hline & & & 5 & $1752 / 0 / 0$ & 132 & - & $\checkmark$ & - \\
\hline & & & 6 & $1724 / 0 / 0$ & 135 & - & $\checkmark$ & - \\
\hline & & & 7 & $7438 /-/-$ & - & $\checkmark$ & - & - \\
\hline \multirow{6}{*}{ b09_gray_onehot } & \multirow{6}{*}{$3 / 1$} & \multirow{6}{*}{$28 / 30$} & 1 & $36846 / 234 / 27462$ & 137 & - & - & $\checkmark$ \\
\hline & & & 2 & $37176 / 240 / 27624$ & 137 & - & - & $\checkmark$ \\
\hline & & & 3 & $27641 / 125 / 17918$ & 137 & - & - & $\checkmark$ \\
\hline & & & 4 & $8381 / 38 / 7048$ & 232 & - & - & $\checkmark$ \\
\hline & & & 5 & $16232 /-/-$ & - & $\checkmark$ & - & - \\
\hline & & & 6 & $12604 / 79 / 12036$ & 232 & - & - & $\checkmark$ \\
\hline & & & 1 & $3150 / 0 / 0$ & 153 & - & $\checkmark$ & - \\
\hline & & & 2 & $8345 / 0 / 0$ & 153 & - & - & $\checkmark$ \\
\hline & & & 3 & $17261 / 298 / 13166$ & 184 & - & - & $\checkmark$ \\
\hline b10_gray_onehot & $13 / 6$ & $17 / 24$ & 4 & $4575 / 0 / 0$ & 153 & - & $\checkmark$ & - \\
\hline & & & 5 & $17261 / 298 / 13166$ & 184 & - & - & $\checkmark$ \\
\hline & & & 6 & $8349 / 0 / 0$ & 184 & - & $\checkmark$ & - \\
\hline & & & 1 & $24534 /-/-$ & - & $\checkmark$ & - & - \\
\hline b13_gray_onehot & $4 / 10$ & $29 / 34$ & 2 & $26550 /-/-$ & - & $\checkmark$ & - & - \\
\hline & & & 1 & $63450 / 0 / 0$ & 384 & - & $\checkmark$ & - \\
\hline & & & 2 & $26399 / 0 / 0$ & 384 & - & $\checkmark$ & - \\
\hline T1_gray_onehot & $30 / 20$ & $66 / 66$ & 3 & $63412 / 0 / 0$ & 384 & - & $\checkmark$ & - \\
\hline & & & 4 & $171075 / 0 / 0$ & 282 & - & $\checkmark$ & - \\
\hline
\end{tabular}

N.E. $=$ Not Equivalent; I.C. = Inconclusive 
one of their circuits under verification. The circuit with Trojan is considered to be suspect circuit and the other circuit is known as spec circuit. The two circuits differ drastically in the number of gates, number of flip-flops, etc. The second column represents the number of input/output ports of each of the circuits. The third column shows the number of flip-flops in the two circuits. Since different state encodings (gray vs. one-hot) were used, the number of flop-flops differ. The fourth column represents the Trojan index. We tabulate the true inductive invariants (two-node same-timeframe, two-node cross-timeframe and three-node invariants) in the fifth column. Two-node inductive invariants were computed among all the signals and state variables, whereas three-node invariants were computed only for state variables. The sixth column shows the number of suspicious signals. In the seventh column, we present the result of sequential equivalence checking. It consists of three sub-columns where the three cases whether the circuits are declared equivalent or inequivalent, or the invariants are insufficient to prove equivalence are included.

For each Trojan insertion cases in Table 3.1, the small circuits b01 and b02 were equivalent when proven using true invariants involving two-node and three-node invariants. But for our study purpose, we limited the number of three-node invariants by considering just flip-flop relations. As a result, the SEC results were inconclusive for which we could use our Trojan detection method.

Next, we have summarized our malicious signal detection results in Table 3.2. For each benchmark, the second column gives the Trojan insertion index. The third column shows the total number of suspicious signals considered in the suspect circuit. In the fourth 
Table 3.2: Results for Malicious Insertion Detection for Hard SEC Benchmark Circuits

\begin{tabular}{|c|c|c|c|c|c|c|c|c|}
\hline \multirow[b]{2}{*}{ Name of Circuit } & \multirow{2}{*}{$\begin{array}{c}\text { Trojan } \\
\#\end{array}$} & \multirow{2}{*}{$\begin{array}{c}\text { \# Sus } \\
\text { Signals }\end{array}$} & \multicolumn{3}{|c|}{ Step 1} & \multicolumn{3}{|c|}{ Step 2} \\
\hline & & & SAT & UNSAT & $\begin{array}{l}\text { Run } \\
\text { Time(s) }\end{array}$ & SAT & UNSAT & $\begin{array}{l}\text { Run } \\
\text { Time(s) }\end{array}$ \\
\hline \multirow{5}{*}{ b01_gray_onehot } & 1 & 40 & 40 & $\overline{0}$ & 0.31 & 31 & $\overline{99}$ & 0.03 \\
\hline & 2 & 40 & 40 & 0 & 0.28 & 31 & 9 & 0.04 \\
\hline & 3 & 40 & 39 & 1 & 0.59 & 28 & 11 & 0.06 \\
\hline & 4 & 40 & 40 & 0 & 0.30 & 31 & 9 & 0.07 \\
\hline & 5 & 42 & 42 & 0 & 0.19 & 20 & 22 & 0.05 \\
\hline \multirow{3}{*}{ b02_gray_onehot } & 1 & 16 & 16 & 0 & 0.04 & 16 & 0 & 0.012 \\
\hline & 2 & 17 & 16 & 1 & 0.03 & 7 & 9 & 0.004 \\
\hline & 3 & 17 & 16 & 1 & 0.04 & 6 & 10 & 0.004 \\
\hline \multirow{2}{*}{ b03_gray_onehot } & 1 & - & - & - & - & - & - & - \\
\hline & 2 & - & - & - & - & - & - & - \\
\hline \multirow[b]{2}{*}{ b06_gray_onehot } & 1 & - & - & - & - & - & - & - \\
\hline & 2 & - & - & - & - & - & - & - \\
\hline \multirow{7}{*}{ b08_gray_onehot } & 1 & 132 & 4 & 128 & 39.39 & 0 & 4 & 0 \\
\hline & 2 & 132 & 50 & 82 & 14.15 & 18 & 32 & 0.14 \\
\hline & 3 & 132 & 5 & 127 & 37.50 & 0 & 5 & 0.004 \\
\hline & 4 & 135 & 64 & 71 & 13.72 & 0 & 64 & 0.08 \\
\hline & 5 & 132 & 42 & 90 & 15.70 & 0 & 42 & 0.02 \\
\hline & 6 & 135 & 66 & 69 & 47.27 & 44 & 22 & 0.44 \\
\hline & 7 & - & - & - & - & - & - & - \\
\hline \multirow{6}{*}{ b09_gray_onehot } & 1 & 137 & 112 & 25 & 348.39 & 57 & 55 & 2.04 \\
\hline & 2 & 137 & 113 & 24 & 355.96 & 56 & 57 & 2.06 \\
\hline & 3 & 137 & 125 & 12 & 583.73 & 59 & 66 & 2.44 \\
\hline & 4 & 232 & 180 & 52 & 1606.13 & 72 & 108 & 0.90 \\
\hline & 5 & - & - & - & - & - & - & - \\
\hline & 6 & 232 & 165 & 67 & 4616.99 & 107 & 58 & 4.16 \\
\hline \multirow{6}{*}{ b10_gray_onehot } & 1 & 153 & 123 & 30 & 535.92 & 79 & 44 & 0.45 \\
\hline & 2 & 153 & 148 & 5 & 713.98 & 141 & 7 & 1.93 \\
\hline & 3 & 184 & 176 & 8 & 654.92 & 90 & 86 & 1.46 \\
\hline & 4 & 153 & 125 & 28 & 361.58 & 72 & 53 & 0.55 \\
\hline & 5 & 184 & 176 & 8 & 686.11 & 92 & 84 & 1.51 \\
\hline & 6 & 184 & 134 & 50 & 372.50 & 66 & 68 & 0.54 \\
\hline \multirow{2}{*}{ b13_gray_onehot } & 1 & - & - & - & - & - & - & - \\
\hline & 2 & - & - & - & - & - & - & - \\
\hline \multirow{4}{*}{ T1_gray_onehot } & 1 & 384 & 62 & 322 & 2454.82 & 58 & 4 & 3.23 \\
\hline & 2 & 384 & 52 & 332 & 803.65 & 36 & 16 & 1.78 \\
\hline & 3 & 384 & 63 & 321 & 2434.03 & 58 & 5 & 3.32 \\
\hline & 4 & 282 & 14 & 268 & 3751.35 & 10 & 4 & 1.20 \\
\hline
\end{tabular}


column, we tabulate the results of the SAT solver in the test generator which is divided into three sub-columns. The first sub-column represents the number of suspicious signals which are declared SAT and thus eligible for counterexample-guided equivalence checking Counterexample Guided Equivalence Checking (CGEC). The second sub-column represents the number of UNSAT suspicious signals. The properties of these UNSAT signals cannot be declared. In the third sub-column, we report total time taken by test generation step to solve all the suspicious signals in seconds. The last multi-column shows the decisions made by SAT solver in the second step, CGEC. It is also divided into three sub-columns. The first sub-column presents the number of SAT suspicious signals which indicates that the spec circuit can produce the same behavior as the sus circuit under the sensitization constraint from step 1, whereas the second sub-column represents the number of UNSAT suspicious signals. Since the two circuits are not equivalent, these signals are declared as the most likely Trojan signals. The last sub-column shows the total time taken by CGEC step to declare suspicious signals in seconds.

The circuits containing alterations were equivalent for all the considered cases in b03 and b06, thus there exist no malicious Trojan. Many alterations in other circuits, e.g. Trojan \#7 of b08, Trojan \#5 of b09, and Trojans \#1 and \#2 of b13 also resulted equivalent during SEC. Therefore, such insertions will not harm the circuit and are benign. Consider insertion \#1 of b09. SEC distinguished spec and suspect circuits. Out of 137 suspicious signals identified for this Trojan, 25 signals failed to activate and propagate at the primary output in test generation step. Counterexample guided Equivalence Checking could declare 55 suspicious 
signals as most likely malicious signals which is 12.14 percent of the total (453) signals in the suspect circuit. Test generation method took 348 seconds whereas CGEC took just 2 seconds. We found the Trojan payload signal among the 55 most-likely malicious signals. As this percentage goes up, it would be very difficult to handle the large number of most-likely signals for large circuit. Therefore, we need to improvise our method by developing a ranking mechanism such that it can narrow down very small percentage of the signal set.

Let us consider insertion \#3 of benchmark T1. This insertion was made by an addition of an inverter at first input of an AND gate. Out of 384 suspicious signals, only 5 were declared most likely malicious which is $0.81 \%$ of total signals in the suspect circuit. All five of them were in the fanout cone of the actual Trojan signal!

Experimental results show that our methodology is effective in narrowing down the number of suspicious signals for many circuits to get most likely malicious signals in the untrusted designs. The Trojan region isolation techniques should look into these signals and identify the relationships between them to narrow down the Trojan location in the circuit.

\subsection{Limitations of the Approach}

The proposed Trojan detection method is limited by the availability of a trusted spec circuit to compare suspect circuit with. Our method is useful to be applied before fabrication step to detect malicious signals in the circuit. But any malicious inclusions in the fabrication phase needs to be detected using various post-silicon Trojan detection techniques. However, some 
types of malicious inclusions in the design stage do not show their activation effect to the outputs. Instead, they leak secret information via side channel leakage or electromagnetic radiations. For detection of such non-functional effects, other alternative methods should be employed.

\subsection{Summary}

In this chapter, we proposed a new Trojan detection methodology in the untrusted thirdparty IP. If the existing equivalence checking cannot prove the suspect and spec circuits to be equivalent, we proceed with our two-step technique to identify the malicious signals in the suspect circuit. The first step involves a light-weight test generation method to activate the suspicious signal and propagate the activation effect to the primary output with the help of powerful invariants. The counterexample obtained in this step and the invariants are used to constrain the search space of equivalence checking in the second step. The use of invariants in both steps make our method very effective by constraining the search space of the SAT solver. 


\section{Chapter 4}

\section{Strengthened Trojan Detection}

\section{Method}

This chapter presents our additional contribution that focuses on making the counterexample generated during test generation more effective. Specifically, we compute the counterexample that witnesses the suspicious behavior of the propagated output in the suspect circuit. We also relax the input vector sequence obtained from the counterexample to prune some of the suspicious signals during equivalence checking by increasing the search space. Finally, we rank the group of most-likely malicious signals to make the search for the Trojan location easier. 


\subsection{Motivation}

The malicious signal detection method proposed in Chapter 3 generates the counterexample during test generation and uses it to reduce the search space during the constrained sequential equivalence checking. In both steps, true invariants are added to further constrain the search space to avoid the illegal states. The strength depends on the counterexample generated, which includes the suspect signal activation path and the input vector sequence. Even if the suspect signal $S_{i}$ is malicious, the propagated value at the primary output through a sensitization path during test generation needs to be malicious for at least one input vector sequence. Therefore, the accuracy of the Trojan detection method can be enhanced by selecting that counterexample in which the propagated effect of the activated suspicious signal is evident to be distinguishable from the output behavior of the genuine circuit.

For a suspicious signal $S_{i}$, the input vector sequence that witnesses the propagation of the activation effect to the primary output is, generally, not the only input vector that lead to the same node assignments in the sensitized path. Therefore, we can relax the bits in the input vector whose values, if flipped, do not alter the node assignments in the sensitization path. Thus, relaxed bit vector represents multiple counterexamples for the same value assignments in sensitized path.

Moreover, the number of most-likely malicious signals declared by the Trojan detection method may be large especially if the circuit is large. This makes the Trojan isolation methods difficult to handle these many number of signals, successfully identify the Trojan 
location in the circuit and correct the circuit behavior. As a result, the development of a ranking algorithm would be extremely helpful to handle large numbers of candidate signals. In particular, a ranking scheme that can tell the stealthy nature of the signal would be very useful.

\subsection{Modified Test Generation}

When a signal $S_{i}$ is activated in the suspect circuit, there may exist multiple paths through which the activation effect is propagated to the primary output without being blocked by the rest of the signals in the circuit. If it is an ordinary signal, the sensitized output from the activation of the suspicious signal would not be harmful. If it is a malicious signal, there may be an alternate path to the same or a different primary output where the propagated effect is malicious instead. To make sure the propagated effect is ordinary (non-malicious), we need to verify that this output behavior against the output of the spec circuit for the corresponding reachable state. Therefore, the test generation method described in Section 3.3.1 can be strengthened by carefully selecting that sensitized path with node value instances for which there exists at least one test vector that can distinguish the behavior of the two circuits (suspect and spec circuits) at the propagated primary output.

After a counterexample is generated using invariant based test generation, we check the output behavior of a miter circuit using a copy of the suspect circuit and a copy of the spec circuit. We unroll the miter for $K^{\prime}+k$ timeframes and use the true invariants in both circuits 
at all timeframes to restrict the state space. We place the node value assignments in the sensitized path only in the suspect circuit. Using the input vector sequence of the counterexample, we check if there exists an initial state pair (and additional input assignments) from which the output pairs corresponding to the sensitized output of the suspect circuit can be differentiated from the corresponding output of the spec circuit. If there exists no state and input vector that can differentiate the two circuits, we find another counterexample using the test generation method and iterate.

Algorithm 1 describes the procedure to check the output behavior of the propagated output due to activation of signal $S_{i}$. The suspicious signal $S_{i}$ gets activated at timeframe $K^{\prime}$ and its propagation effect reaches the primary output of suspect circuit at timeframe $K^{\prime}+k-1$. For any timeframe $t f, I_{t f}, I n v_{t f}$ and $O_{t f}$ represent the primary input, true invariant list and final miter output, respectively. Input vectors added as constraint are the same as the input vectors obtained as counterexample obtained from Section 3.3.1. Output $O_{0}$ to $O_{K^{\prime}+k-2}$ are forced to logic ' 0 ' whereas $O_{K^{\prime}+k-1}$ is forced to logic ' 1 '. This essentially ensures that the two circuits are not distinguished in a time-frame before the final (right-most) time-frame. In this last timeframe, the XOR output of the primary output pairs corresponding to the primary output of the suspect circuit in the sensitized path are forced to logic ' 1 '. The propositional formula consisting of the unrolled miter along with the constraints is given to a SAT solver to check if the output of the two circuits can be distinguished, i.e., if there exists a state pair from which the given input vector sequence can distinguish the two circuits.

If the SAT solver returns satisfiable, the propagated value of the activation of $S_{i}$ at the 


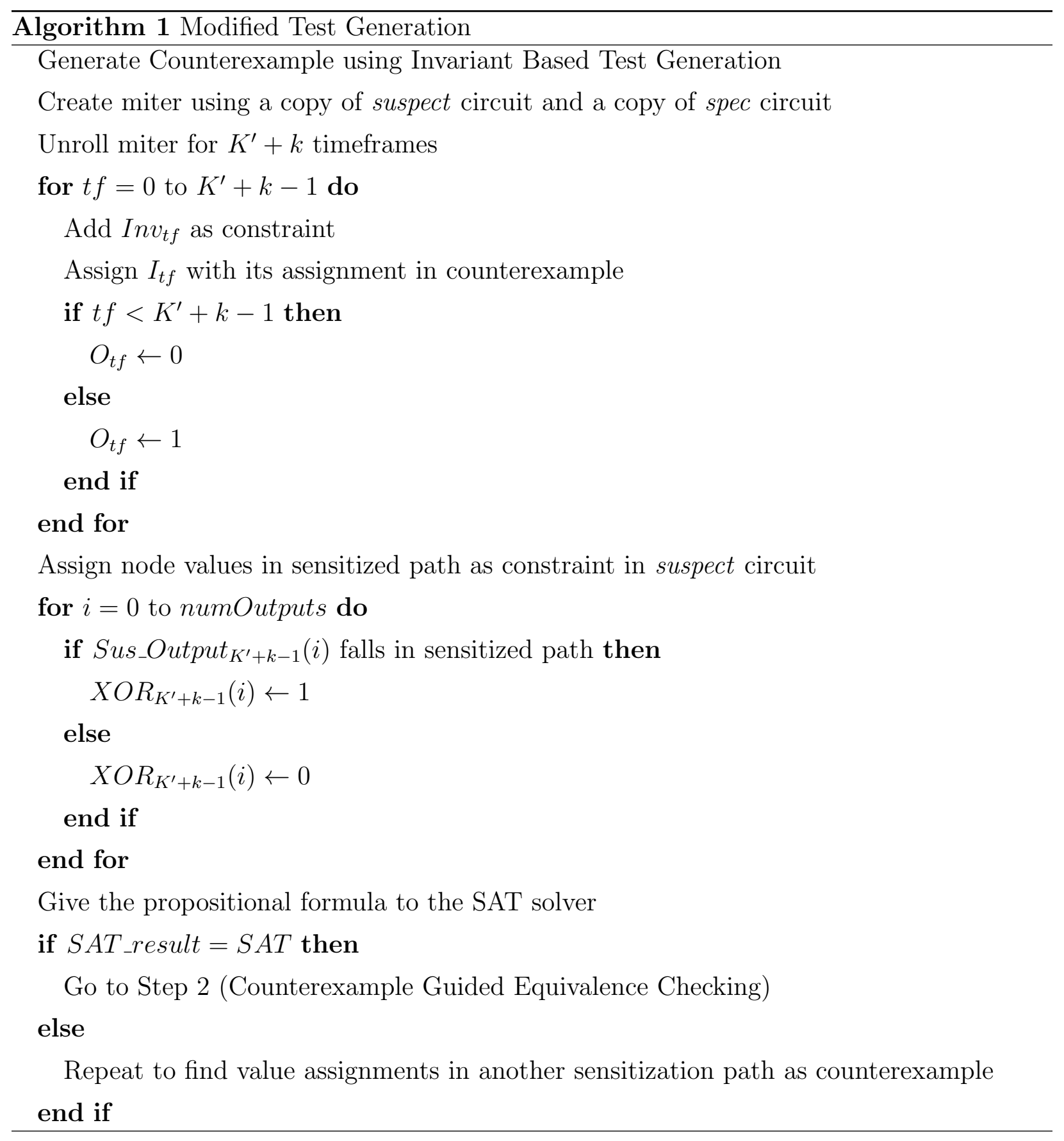

primary output is likely to be malicious, since we cannot guarantee the reachability of the initial state pair. So, we proceed with the counterexample guided equivalence checking method described in Section 3.3.2. On the other hand, if the SAT solver returns unsatisfiable, 
we repeat the test generation process by finding another sensitization path where the node assignments act as witness to suspicious signal activation and propagation.

Because of the selection of better counterexample, the chance of the final signal list to contain the malicious Trojan signal will be higher than the method discussed in the previous

chapter. But we cannot get rid of all non-malicious signals because of the possibility of the unreachable states during the process. Moreover, this cannot be achieved without a cost. Since the extra step needs to be performed on the top of invariant based test generation iteratively for each suspect signal, the overall run time of this method can be significantly higher which may not be tolerable especially for large circuits.

\subsection{Relaxed Input Vector as Constraint}

The input sequence from the counterexample is one of the counterexample assignments that activates and propagates a candidate suspicious signal. We may obtain the same activation and propagation path with a different starting state or a different input sequence. For the same state assignments in the counterexample, we can relax some of the input bits and get the same sensitized path assignment by replacing deterministic value either logic ' 0 ' or logic ' 1 ' by a don't care. If we flip the input and obtain the same activation and propagation path assignment, that input assignment is not necessary in the value assignments at the sensitized path and can be left unconstrained. When all the relaxable bits are indeed relaxed, the rest of the bits in the input vector play necessary roles in restricting the search space during SAT 


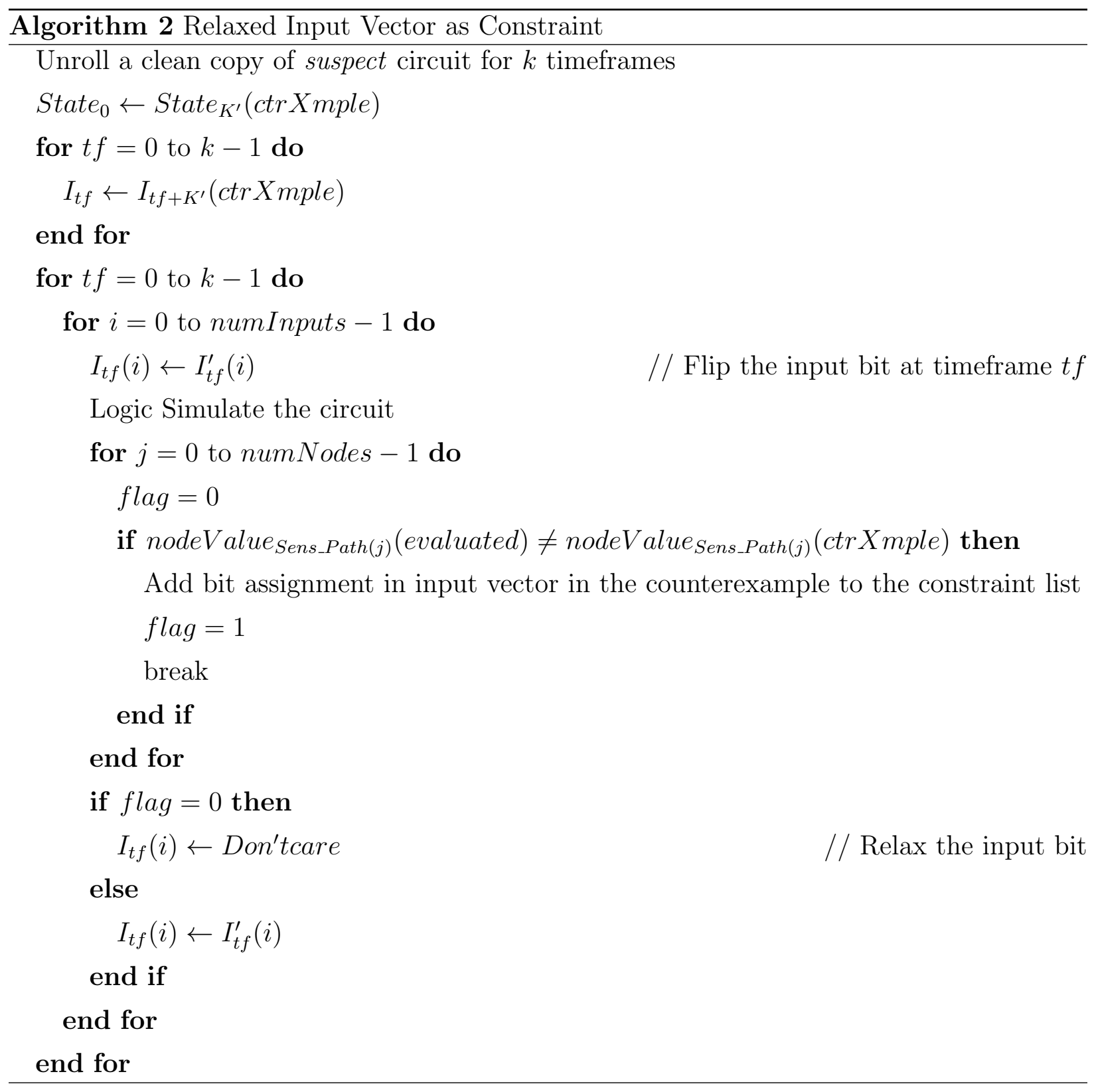

solving and their assignments are added as constraints.

Algorithm 2 illustrates the procedure to relax the counterexample inputs and generates the input vector constraints for counterexample guided equivalence checking (CGEC). We take a single copy of the suspect circuit and unroll it for $k$ timeframes. We activate the suspicious 
signal $S_{i}$ at timeframe 0 and propagate the activation to the primary output at timeframe $k$. We assign the pseudo-primary inputs with the flip-flop assignment at timeframe $K^{\prime}$. We also assign the input vector at timeframe $t f$ with the counterexample assignment at timeframe $t f+K^{\prime}$. We complement an input bit, logic simulate and check if any of the nodes in suspicious signal activation and propagation path is affected.

In this algorithm, numNodes is the total number of nodes in the sensitization path. For any value of $j$ where $(0 \leq j \leq$ numNodes -1$)$, we check if the evaluated value at $\operatorname{Sens\_ Path}(j)$ during logic simulation is different from the counterexample value, and if yes, the bit assignment in the input vector is very crucial in activating and propagating the suspicious signal, and therefore we add the input bit assignment of the input vector in the counterexample to the constraint list to be added in CGEC.

Since this method reduces the number of constraints during SAT solving, the number of satisfaible solutions returned by the SAT solver would be higher compared to that in the previous chapter. But the increase in the search space will increase the SAT solving time to perform CGEC.

\subsection{Ranking of Stealthiness of Malicious Signals}

Consider the set of $M$ most-likely malicious signals declared by our Trojan detection algorithm be $\left\{m_{1}, m_{2}, m_{3} \ldots m_{M}\right\}$. These signals can be ranked based on the importance of the signal in activation and propagation of other most-likely malicious signals during malicious 
signal detection.

In the counterexample involving the activation and propagation of a signal $m_{i}$, where $(1 \leq$ $i \leq M)$, we check the value assignments of the most-likely malicious signals in the sensitized path. We keep track of the activation of these signals in the counterexamples by giving score $S$ core $_{i}$ to each signal $S_{i}$. Since each signal $m_{i}$ is definitely activated in the counterexample related to its activation, its score is at least one. If $m_{i}$ gets activated at least once in the sensitized path of the activation of another signal $m_{j}$, $(1 \leq j \leq M)$, the score of $m_{i}$ is incremented by 1 . Therefore, the maximum value of the score for $m_{i}$ can be $M$ if we observe its activation in the sensitized path for all the $M$ counterexamples.

The ranking of the most-likely malicious signal $S_{i}$ can be determined using its score. A higher score indicates a lower rank. We should keep in mind that out of the malicious signals list, none of the signals may be the malicious signal. This ranking method does not say about the likeliness of one malicious signal among all candidates. Instead, it tells about the stealthiness of the Trojan signal.

If the rank of a signal $m_{i}$ is low, it means that the signal falls in many sensitization paths of the other suspicious signals and thus, the activation of these other signals may be related on the activation of $m_{i}$. As a result, the probability of the detection of $m_{i}$ would be higher compared to other signals. In other words, this signal $m_{i}$, if truly malicious, is less stealthy as it has more potential counterexamples. On the other hand, if the rank of a stealthy signal is high, the activation of other signals is less likely to depend on its activation. Therefore, if it is a true malicious signal, the input sequence that activates and propagates its effect 
is harder to be achieved among all the candidate suspects. This makes this signal more stealthy compared to other lower ranked signals.

\subsection{Experimental Results}

The algorithms described in this chapter were implemented to target a suite of hard-to-verify SEC benchmarks constructed from gray and one hot encodings of ITC99 benchmark circuits where the two circuits had different number of I/O and gate counts with very few or no internal equivalent nodes. Minute hard Trojans were inserted at one of the original circuits by adding inverter(s), deleting inverter(s) or changing the gate type.

Table 4.1 presents the result of modified malicious signal detection method which involves improved test generation and the use of relaxed input vector method combined with ranking method of the final signal list. Column 1 represents the benchmark circuits. Column 2 presents the Trojan index for inserted in one of the circuits of each miter. Column 3 tabulates the total number of suspicious signals considered for the Trojan detection. Column 4 and 5 illustrate the result of the modified test generation method explained in Section 4.2. Column 6 and 7 illustrate the result of counterexample guided equivalence checking using the input vector relaxation method discussed in Section 4.3. The signals in Column 4 is the sum of the signals in the columns 6 and 7 . The signals represented by Column 7 are the most-likely malicious signals whereas the behavior of signals represented by columns 5 and 6 cannot be determined. The column 8 presents the percentage of most-likely malicious signals with 
Table 4.1: Results for Improved Test Generation and Vector Relaxation

\begin{tabular}{|c|c|c|c|c|c|c|c|c|}
\hline \multirow[b]{2}{*}{ Name of Circuit } & \multirow{2}{*}{$\begin{array}{c}\text { Trojan } \\
\#\end{array}$} & \multirow{2}{*}{$\begin{array}{l}\text { \# Sus. } \\
\text { Signals }\end{array}$} & \multicolumn{2}{|c|}{ Test Gen } & \multicolumn{2}{|c|}{ CGEC } & \multirow{2}{*}{$\begin{array}{c}\text { \% Mali- } \\
\text { cious }\end{array}$} & \multirow[b]{2}{*}{ Rank } \\
\hline & & & SAT & UNSAT & SAT & UNSAT & & \\
\hline \multirow{6}{*}{ b08_gray_onehot } & 1 & 132 & 34 & 98 & 13 & 21 & 7.6 & 1 \\
\hline & 2 & 135 & 24 & 111 & 11 & 13 & 4.7 & 1 \\
\hline & 3 & 132 & 26 & 106 & 0 & 26 & 9.4 & 23 \\
\hline & 4 & 135 & 21 & 114 & 0 & 21 & 7.6 & 19 \\
\hline & 5 & 135 & 1 & 134 & 0 & 1 & 0.36 & - \\
\hline & 6 & 132 & 22 & 110 & 20 & 2 & 0.72 & 1 \\
\hline \multirow{3}{*}{ b09_gray_onehot } & 1 & 232 & 90 & 142 & 29 & 61 & 14.3 & 59 \\
\hline & 2 & 232 & 6 & 226 & 5 & 1 & 0.22 & 1 \\
\hline & 3 & 232 & 25 & 207 & 10 & 15 & 3.3 & 15 \\
\hline \multirow{5}{*}{ b10_gray_onehot } & 1 & 153 & 22 & 131 & 19 & 3 & 0.93 & - \\
\hline & 2 & 184 & 59 & 125 & 59 & 0 & 0 & - \\
\hline & 3 & 153 & 22 & 131 & 18 & 4 & 1.24 & 1 \\
\hline & 4 & 184 & 82 & 102 & 76 & 26 & 8.07 & 13 \\
\hline & 5 & 153 & 46 & 107 & 80 & 27 & 8.38 & 9 \\
\hline \multirow{4}{*}{ T1_gray_onehot } & 1 & 384 & 20 & 364 & 14 & 6 & 0.98 & 1 \\
\hline & 2 & 384 & 26 & 358 & 0 & 26 & 4.23 & 23 \\
\hline & 3 & 384 & 42 & 342 & 0 & 42 & 6.8 & 37 \\
\hline & 4 & 282 & 21 & 261 & 0 & 21 & 3.4 & 19 \\
\hline
\end{tabular}

respect to the total number of signals in the circuit. This percentage should be very low especially in case of large circuit in order to locate the exact Trojan location in the circuit. The last column tabulates the ranking of the actual inserted Trojan in the suspect circuit.

Consider example, Trojan \# 1 for the circuit T1. 6 signals out of 384 suspicious signals were found to be most-likely malicious which is $0.98 \%$ of total signals (614) in the suspect circuit. The rank of the actual Trojan is found to be 1 in this case.

Consider another example, Trojan \# 3 for the circuit b08. Out of 132 suspicious signals, the total number of most-likely malicious signals is found to be 26 as represented by Column 7 . 
This number is $9.4 \%$ of the total signals (276) in the suspect circuit. The inserted Trojan signal was ranked almost the least which means that the stealthiness of the Trojan is low. This shows that as a Trojan is less stealthy, more suspicious signals accompany the true malicious signal.

The effectiveness of the strengthening method was evaluated by comparing the signals involved in test generation and equivalence checking. In an evaluation involving an insertion in the circuit b10, the earlier technique (the technique discussed in Chapter 2) returned 70 signals as most-likely malicious signals whereas this method returned 30 signals even though the actual trojan signal was found in both lists. However, the SAT solving time for previous method was 306.82 seconds for Case 1 and 0.44 seconds for Case 2, whereas that for the strengthened method was 18527.1 seconds for Step 1 and 0.47 seconds for Step 2. Therefore, even though strengthening methods led to fewer and more intelligent signals in the final signal list, the modified test generation method is found to be very time-consuming whereas there is very little overhead in run time because of the input vector relaxation method.

\subsection{Conclusion}

In this chapter, we modify the malicious signal detection strategy explained in the previous chapter. The test generation technique is modified to make the counterexample more reliable. The input vector in the counterexample is relaxed that gives same activation and propagation path. The malicious signal ranking mechanism can be used to check the stealthiness of the 
signal of interest. Experimental results show that the modified technique intelligently gives us the small set of most-likely malicious with significant run time overhead. It also helps to determine the stealthiness of a candidate Trojan. 


\section{Chapter 5}

\section{Conclusion and Future Work}

In this thesis, we have proposed a novel technique that combines constrained sequential equivalence checking and test generation method to detect malicious signals in the IP cores received from an untrusted third party. We start with creating a miter circuit using the suspect circuit and the reference spec circuit, identify a set of suspicious signals in the suspect circuit and apply our two-step Trojan detection methodology. The first step is invariant based test generation where we find counterexample that activates the suspicious signal and propagates its effect to the primary output. The second step is counterexample guided equivalence checking where we use counterexample generated from previous step to check the behavior of spec circuit when there is activation effect of suspicious signal in the suspect circuit. The counterexample used is the node assignments in the sensitized path which propagate the signal activation to the primary output in the suspect circuit. The true inductive invariants are applied in both steps to prune the large illegal state space. Experimental 
results on hard SEC instances of ITC99 benchmark circuits demonstrate that our method can either ensure that the suspected design is free from functional effects of any malicious changes or return a small set of the most likely malicious signals when we cannot guarantee the trust of third-party design. Thus, this methodology is very handy in ensuring the trust in hardware designs.

We have also proposed the modified version of malicious signal detection methodology where we strengthen the counterexample generated in the test generation before using it for counterexample guided equivalence checking. We find that sensitized path assignment for which the output pairs corresponding to sensitized output can be distinguishable for the input vector sequence. We also relax the input sequence that can generate the same node assignments in the sensitized path. Finally, we have proposed the ranking mechanism for the most-likely malicious signals that show the stealthiness of the final signal list.

In the future, we want explore the use of multiple counterexamples to make the scheme more efficient. We would also like to develop the different type of ranking mechanism which can rank the suspicious signals according to the malicious behavior which can narrow down the malicious signal search. In addition, we would like to develop the Trojan isolation mechanism together with this approach to successfully locate the malicious region in the circuit. 


\section{Bibliography}

[1] G. E. Moore, Cramming More Components onto Integrated Circuits, Electronics, Vol. 38, No. 8, April 1965, pp. 114-117.

[2] S. Adee, The Hunt for the Kill Switch, IEEE Spectrum, Vol. 45, No. 5, 2008, pp. 34-39.

[3] DARPA, Trust in Integrated Circuits(TIC), http://www.dtic.mil/cgibin/GetTRDoc?AD=ADA503809, accessed 14 July, 2012.

[4] H. Bar-El, H. Choukri, D. Naccache, M. Tunstall, and C. Whelan, The Sorcerer's Apprentice Guide to Fault Attacks, in Proceedings of the IEEE, 2006, pp. 370-382.

[5] P. Kocher, J. Jaffe, and B. Jun, Differential Power Analysis, in Proc. of 19th International Advances in Cryptology Conference CRYPTO, August 1999, pp. 388-397.

[6] M. G. Kuhn, Security Limits for Compromising Emanations, in Proc. of Cryptographic Hardware and Embedded Systems(CHES), Vol. 3659 of LNCS, Springer, 2005.

[7] K. Gandol, C. Mourtel, and F. Olivier, Electromagnetic analysis: Concrete results, Cryptographic Hardware and Embedded Systems (CHES), 2001, pp. 251-261. 
[8] P. Kocher, Timing Attacks on Implementations of Diffie-Hellman, RSA, DSS, and Other Systems, CRYPTO, 1996, pp.104-113.

[9] T.S. Messerges, E.A. Dabbish and R.H. Sloan, Investigations of Power Analysis Attacks on Smartcards, in Proc. USENIX Workshop Smartcard Technology, May 1999, pp. 151161.

[10] Trusted Computing Group, Incorporated, TCG specication architecture overview, 2007.

[11] Y. Ding, Y. Du, Z. Gao, Y. Chen, F. Bai, X. Cai, Design of watermarking systems for IP protection, in International Conference on Solid-State and Integrated-Circuit Technology, Oct. 2008, pp. 1893-1896.

[12] R. Maes, D. Schellekens, P. Tuyls, and I. Verbauwhede, Analysis and design of active IC metering schemes, in HOST, 2009, pp. 74-81.

[13] E. Castillo and U. Meyer-Baese, A. Garcia, L. Parrilla, and A. Lloris, IPP@HDL: Efficient Intellectual Property Protection Scheme for IP Cores, in IEEE Transactions on VLSI, Vol. 15, No. 5, 2007, pp. 578-590.

[14] F. Bai, Z. Gao, Y. Xu, and X. Cai, A Watermarking Technique for Hard IP Protection in Full-custom IC Design, in International Conference on Communications, Circuits and Systems (ICCCAS), July 2007, pp. 1177-1180. 
[15] Y. Du, Y. Ding, Y. Chen, and Z. Gao, IP protection platform based on watermarking technique, in Proc. of the 2009 10th Int'l Symp. on Quality of Electronic Design, 2009, pp. $287-290$.

[16] A.B. Kahng, J. Lach, W.H. Mangione-Smith, S. Mantik, I.L. Markov,M. Potkonjak, P. Tucker, H. Wang, and G. Wolfe, Watermarking Techniques for Intellectual Property Protection, in Proc. 35th ACM/IEEE Design Automation Conference (DAC), June 1999, pp. 776-781.

[17] R. S. Chakraborty and S. Bhunia, Security Through Obscurity: An Approach for Protecting Register Transfer Level Hardware IP, in IEEE International Workshop on Hardware-Oriented Security and Trust, 2009, pp. 96-99.

[18] F. Koushanfar, Provably secure active IC metering techniques for piracy avoidance and digital rights management, in IEEE Transactions on Information Forensics and Security, 2012, pp. 51-63.

[19] G. E. Suh and S. Devadas, Physical unclonable functions for device authentication and secret key generation, in Proceedings of the 44th Design Automation Conference, IEEE, 2007, pp. 9-14.

[20] J. Guajardo, S. S. Kumar, G.-J. Schrijen, and P.Tuyls, Brand and IP protection with physical unclonable functions, in International Symposium on Circuits and Systems, May 2008, pp. 3186-3189. 
[21] J. Guajardo, B. Skoric, P. Tuyls, S. S. Kumar, T. Bel, A. H. M. Blom, and G.-J. Schrijen, Anti-counterfeiting, key distribution, and key storage in an ambient world via physical unclonable functions, ISF, Vol. 11, No. 1, 2009, pp. 19-41.

[22] V. Vivekraja and L. Nazhandali, Circuit-Level Techniques for Reliable Physically Uncloneable Functions, in IEEE International Workshop on Hardware-Oriented Security and Trust, 2009, pp. 30-35.

[23] J. Kumagai, Chip detectives, IEEE Spectrum, Vol. 37, No. 11, Nov. 2000, pp. 43-49.

[24] R. Torrance and D. James, The State-of-the-Art in Semiconductor Reverse Engineering, in Design Automation Conference (DAC), 2011, pp. 333-338.

[25] R. S. Chakraborty and S. Bhunia, RTL hardware IP protection using key-based control and data flow obfuscation, in International Conference on VLSI Design, 2010, pp. 405410.

[26] R. Chakraborty and S. Bhunia, HARPOON: An obfuscation-based SoC design methodology for hardware protection, in IEEE Transactions on Computer-Aided Design Integrated Circuits and Systems, Vol. 28, No. 10, 2009, pp. 1493-1502.

[27] W. Hu, H. Nguyen, and M. S. Hsiao, Sufficiency-based filtering of invariants for Sequential Equivalence Checking, in Proceedings of IEEE High Level Design Validation and Test Workshop, 2011, pp. 1-8. 
[28] M. Banga, M. Chandrasekar, L. Fang and M. Hsiao, Guided test generation for isolation and detection of embedded Trojans in ICs, ACM Great Lake Symposium on Very Large Scale Integration, 2008, pp. 363-366.

[29] M. Banga and M. Hsiao, A Region Based Approach for the Detection of Hardware Trojans, IEEE Int'l Workshop on Hardware-Oriented Security and Trust, 2008, pp. 43-50.

[30] M. Banga and M. Hsiao, Novel Sustained Vector Technique for the Detection of Hardware Trojans, in Proc. Int'l Conf. VLSI design, 2009, pp. 327-332.

[31] R. Karri, J. Rajendran, K. Rosenfeld, and M. Tehranipoor, Trustworthy hardware: Identifying and classifying hardware Trojans, IEEE Computer, Vol. 43, No. 10, Oct. 2010, pp. 39-46.

[32] J. Rajendran, E. Gavas, J. Jimenez, V. Padman, and R. Karri, Towards a comprehensive and systematic classification of hardware trojans, in Proceedings of IEEE International Symposium on Circuits and Systems, 2010, pp. 1871-1874.

[33] Y. Jin and Y. Makris, Hardware Trojan detection using path delay fingerprint, IEEE Int'1 Workshop on Hardware-Oriented Security and Trust, 2008, pp. 51-57.

[34] F. Wolff et al., Towards Trojan Free Trusted ICs: Problem Analysis and Detection Scheme, Proc. Design, Automation and Test in Europe Conf. (DATE 08), ACM Press, 2008, pp. 1362-1365. 
[35] X. Wang, M. Tehranipoor, and J. Plusquellic, Detecting Malicious Inclusions in Secure Hardware: Challenges and Solutions, Proc. IEEE Int'l Workshop HardwareOriented Security and Trust (HOST 08), IEEE CS Press, 2008, pp. 15-19.

[36] J.A. Roy, F. Koushanfar, and I.L. Markov, Extended Abstract: Circuit CAD Tools as a Security Threat, Proc. IEEE Workshop Hardware-Oriented Security and Trust (HOST 08), IEEE Press, 2008, pp. 65-66.

[37] E. Love, Y. Jin, and Y. Makris, Enhancing security via provably trustworthy hardware Intellectual Property, in IEEE Int'l Symp. on Hardware-Oriented Security and Trust, 2011, pp. 12-17.

[38] Y. Jin and Y. Makris, Hardware trojans in wireless crypto graphic ICs, IEEE Design and Test of Computers, vol. 27, 2010, pp. 26-35.

[39] D. Agrawal, S. Baktir, D. Karakoyunlu, P. Rohatgi and B. Sunar, Trojan Detection using IC Fingerprinting, IEEE Int'l Symp. on Security \& Privacy, 2007, pp. 296-310.

[40] R. Rad, X. Wang, J. Plusquellic and M. Tehranipoor, Power Supply Signal Calibration Techniques for Improving Detection Resolution to Hardware Trojans, in Proc. of the Int'l Conf. on Computer-Aided Design, 2008, pp. 632-639.

[41] C. Lamech, J. Plusquellic, Trojan Detection based on Delay Variations Measured using a High-Precision, Low-Overhead Embedded Test Structure, IEEE Int'l Symp. on Hardware-Oriented Security and Trust, 2012, pp. 75-82. 
[42] D. Rai and J. Lach, Performance of Delay-Based Trojan Detection Techniques under Parameter Variations, in Proc. of IEEE International Workshop on Hardware-Oriented Security and Trust (HOST), July 2009, pp. 58-65.

[43] R.S. Chakraborty, F. Wolff, S. Paul, C. Papachristou, S. Bhunia, MERO: A Statistical Approach for Hardware Trojan Detection, CHES Workshop, 2009, pp. 396-410.

[44] S. Wei, S. Meguerdichian, and M. Potkonjak, Gate-level characterization: foundations and hardware security applications, DAC, 2010, pp. 222-227.

[45] Y. Alkabani and F. Koushanfar, Extended Abstract: Designers Hardware Trojan Horse, in Proc. IEEE International Workshop Hardware-Oriented Security and Trust (HOST), 2008, pp. 82-83.

[46] Y. Jin, N. Kupp, and M. Makris, DFTT: Design for Trojan test, in Proc. IEEE Int'l Conf. Electronics Circuits and Systems, 2010, pp. 1175-1178.

[47] M. Hicks, M. Finnicum, S. T. King, M. M. K. Martin, and J. M. Smith, Overcoming an untrusted computing base: Detecting and removing malicious hardware automatically, in Proceedings of the IEEE Symposium on Security and Privacy, 2010, pp. 159-172.

[48] E. Love, Y. Jin, Y. Makris, Proof-Carrying Hardware Intellectual Property: A Pathway to Trusted Module Acquisition, in IEEE Transactions on Information Forensics and Security, Feb 2012, pp. 25-40. 
[49] M. Potkonjak, Synthesis of trustable ICs using untrusted CAD tools, in Proc. Design Automation Conference (DAC), 2010, pp. 633-634.

[50] X. Zhang and M. Tehranipoor, Case study: Detecting Hardware Trojans in third-party digital IP cores, in IEEE Int'1 Symp. on Hardware-Oriented Security and Trust, June 2011, pp. 67-70.

[51] T. Reece, D. B. Limbrick, W. H. Robinson, Design Comparison to Identify Malicious Hardware in External Intellectual Property, IEEE Int'l Conf. on Trust, Security and Privacy in Computing and Communications, November 2011, pp. 639-646.

[52] M. Banga and M. S. Hsiao, Trusted RTL: Trojan detection methodology in pre-silicon designs, in Proc. IEEE Hardware-Oriented Security \& Trust Symp., 2010, pp. 56-59.

[53] J. Zhao, M. Rudnick, and J. Patel, Static logic implication with application to fast redundancy identication, in Proc. 15th IEEE VLSI Test Symposium, 1997, pp. 288-293.

[54] J. Zhao, J. A. Newquist and J. Patel, A graph traversal based framework for sequential logic implication with an application to c-cycle redundancy identification, in Proc. VLSI Design Conf., 2001, pp. 163-169.

[55] K. Gulrajani and M. S. Hsiao, Multi-node static logic implications for redundancy identification, in Proceedings of the conference on Design, automation and test in Europe, March 2000, pp.729-735. 
[56] S. Cook, The complexity of theorem proving procedures, in Proceedings of ACM Symposium on Theory of Computing, 1971, pp. 151-158.

[57] N. Een, N. Sorensson, An extensible SAT-solver [ver 1.2], 2003.

[58] M. W. Moskewicz, C. F. Maadigan, Y. Zhao, L. Zhang and S. Malik, Chaff: Engineering an Efficient SAT Solver, DAC, 2001, pp. 530-535.

[59] J. P. Marques-Silva and K. A. Sakallah, GRASP: A New Search Algorithm for Satisfiability, in Proceedings of International Conference on Computer-Aided Design, 1996, pp. $220-227$.

[60] M. Davis, G. Logemann, and D. Loveland, A machine program for theorem-proving, Communications of the ACM, Vol. 5, No.7, 1962, pp. 394-397.

[61] A. Biere, A. Cimatti, E. M. Clarke, and Y. Zhu, Symbolic model checking without BDDs, in TACAS, 1999, pp. 193-207.

[62] M. Sheeran, S. Singh, and G. Stalmarck, Checking safety properties using induction and a SAT-solver, in Proceedings of the International Conference on Formal Methods in Computer Aided Design (FMCAD), Vol. 1954 of Lecture Notes in Computer Science, Springer, 2000, pages 108-125.

[63] K. L. McMillan, Applying SAT Methods in Unbounded Symbolic Model Checking, in Proc. Computer Aided Verification, Vol. 2404 of LNCS, 2002, pp. 250-264. 
[64] C. van Eijk, Sequential equivalence checking based on structural similarities, in IEEE Trans. Computer-Aided Design, Volume: 19, Issue: 7, July 2000, pp. 814-819.

[65] S.-Y. Huang, K.-T. Cheng, K.-C. Chen, C.-Y. Huang and F. Brewer, AQUILA: an equivalence checking system for large sequential designs, IEEE Trans. on Computers, Volume: 49 Issue: 5, May 2000, pp. 443-464.

[66] F. Lu and K.-T. Cheng, SEChecker: A sequential equivalence checking framework based on kth invariants, in IEEE Transactions on Very Large Scale Integration (VLSI) Systems, Vol. 17, No. 6, June 2009, pp. 733-746. 